\title{
A Signal Based Triangular Structuring Element for Mathematical Morphological Analysis and Its Application in Rolling Element Bearing Fault Diagnosis
}

\author{
Zhaowen Chen, Ning Gao, Wei Sun, Qiong Chen, Fengying Yan, Xinyu Zhang, \\ Maria Iftikhar, Shiwei Liu, and Zhongqi Ren \\ College of Chemical Engineering, Beijing University of Chemical Technology, Beijing 100029, China \\ Correspondence should be addressed to Wei Sun; sunwei@mail.buct.edu.cn
}

Received 17 August 2013; Accepted 18 February 2014; Published 1 April 2014

Academic Editor: Valder Steffen

Copyright (C) 2014 Zhaowen Chen et al. This is an open access article distributed under the Creative Commons Attribution License, which permits unrestricted use, distribution, and reproduction in any medium, provided the original work is properly cited.

Mathematical morphology $(\mathrm{MM})$ is an efficient nonlinear signal processing tool. It can be adopted to extract fault information from bearing signal according to a structuring element (SE). Since the bearing signal features differ for every unique cause of failure, the SEs should be well tailored to extract the fault feature from a particular signal. In the following, a signal based triangular SE according to the statistics of the magnitude of a vibration signal is proposed, together with associated methodology, which processes the bearing signal by MM analysis based on proposed SE to get the morphology spectrum of a signal. A correlation analysis on morphology spectrum is then employed to obtain the final classification of bearing faults. The classification performance of the proposed method is evaluated by a set of bearing vibration signals with inner race, ball, and outer race faults, respectively. Results show that all faults can be detected clearly and correctly. Compared with a commonly used flat SE, the correlation analysis on morphology spectrum with proposed SE gives better performance at fault diagnosis of bearing, especially the identification of the location of outer race fault and the level of fault severity.

\section{Introduction}

Rolling element bearings are one of the most important and common components in rotating machinery. Their carrying capacity and reliability are essential for the overall machine performance. Therefore the fault diagnosis of rolling element bearing has been studied intensively for the security of mechanical systems [1]. When a fault in one surface of a bearing strikes another surface, a force impulse is generated which excites some vibration response in the bearing and machine system. The vibration response can be obtained and converted into vibration signal. As most information concerning the fault feature is contained in vibration signal, the vibration-based bearing fault diagnosis method has attracted extensive interests from both academia and industry [2, 3]. The vibration signals, usually indirect and nonlinear, are additionally masked by noise. Therefore an accurate signal processing and final diagnosis largely depend on the extraction of feature information from vibration signals.
A number of studies have been conducted on vibration signal processing [4]. The most accepted approach for the demodulation and feature extraction of vibration signal, the envelope analysis (EA) technique $[5,6]$, has been widely used in the detection of mechanical failures since 1980s. However, a prior knowledge of the filtering band is required in EA, which limits its application. In order to overcome this limitation, wavelet transform (WT) has been incorporated into conventional envelope analysis in recent years $[7,8]$. This technique enables simultaneous multiscale decomposition to extract and separate envelopes of repetitively excited mechanical vibrations with different frequency coverage. Although the WT is considered as the best time-frequency analysis and noise reduction method, it is still accompanied with the interference terms, border distortion, and energy leakage, which make the results confusing and difficult for interpretation $[9,10]$. Furthermore, multiscale information incurred a significant computational cost [11]. Empirical mode decomposition (EMD) [12], which has been widely 
used in feature extraction, has been applied to nonlinear and nonstationary signal processing in the last ten years [13]. However, undesired frequency components in results and undesired low amplitude IMFs at the low-frequency region remain unsolved in EMD $[10,14]$.

Mathematical morphology (MM) [15-17] is an efficient nonlinear signal processing tool which has been used for signal processing solely in time domain rather than frequency domain. It can be employed to decompose a signal into several physical components according to the geometric characteristics of a certain SE. Recently the MM has been used as feature extractor and noise filter for fault diagnosis in rotating machinery, for it has better performance at keeping pulse information and rejecting white noise [18-21].

Nikolaou and Antoniadis [22] introduced MM into fault diagnosis of rolling element bearings. They employed a closing operator and a flat (zero) SE with a length of 0.6 times the impulse repetition period for the impulsive periodic signals in fault detection. As pointed out by Dong et al. the SE and its length may not be suited for all situations [23]. They adopted a signal to noise ratio (SNR) to select the optimal length of flat SE when using the MM. Wang et al. [20] proposed an improved morphological filter for the feature extraction of fault frequency, natural frequency, and decay coefficient. They constructed a new SE with an impulse attenuation signal and proposed a new criterion to optimize the SE, but this method requires the prior knowledge of the signals. Recently, multiscale MM [1, 2, 5, 11, 24] has been studied to extract features at different scales. The multiscale MM also gives a better performance at fault detection of rotating machinery, and no prior knowledge is required, but the computation complexity limits its application.

Structuring element (SE), defined by an array of elements with specific values, is a key component in MM analysis, and its geometry has a decisive effect on analysis results [24]. Generally, more feature information can only be extracted when the geometry of a SE is similar to that of a signal. The SE geometry is characterized by three properties: shape, length (number of elements in SE), and height (value of elements in $\mathrm{SE}$ ). SE applied on a $1 \mathrm{D}$ signal can be in various shapes, such as flat, triangular, semicircular, sine, curve, and other polygons. The flat, triangular, and semicircular SEs are good candidates for vibration signal analysis, with triangular SE being the best one among all candidates $[2,18,24]$. In addition to the shape, the length and height of SE should also be considered in practice. Usually more impulse features can be extracted by shorter SE. When a longer SE is used, more noise may be suppressed, while more peaks and impulse information will be lost. Furthermore, the longer and more complex the SE is, the more the computation time to conduct the MM process is [25].

When MM is applied to fault diagnosis of rolling element bearing, the SE is usually selected with experience, for there are few rules or guidelines for the selection. As the flat SEs used previously differ mainly in the selected length scale, the SE's geometry is similar and too simple to extract different features of a signal. In MM analysis, the SE acts as a feature extraction window, and the signal components can be preserved only when component geometries are similar to that of the SE. Since the geometry of three continuous sample sets of a signal can be considered as a generalized triangle, the use of triangular SE with three data points may perform better on the feature extraction of a signal. The triangular SEs are also reported in many previous papers to remove noise and extract more fault features than any other type in vibration signal processing $[18,24,26]$. Besides the shape and length of triangular SE, the height is another key parameter in SE construction and may have an effect on feature extraction results to some extent. As the vibration signals of bearing with inner race, outer race, and ball faults have different characteristics and amplitudes, the SE used for processing those signals should also be different. However, how the height of a SE influences the performance of feature extraction has not been reported yet. This paper proposes a triangular SE with the height corresponding to $3 \sigma$ in the statistics of vibration signal magnitude. The performance of the proposed SE, expected to extract more useful feature information from the original signal, is tested using bearing vibration signals from the Bearing Data Center of Case Western Reserve University (CWRU) [27].

Section 2 of this paper provides a brief review of MM and correlation analysis. Additionally, construction of a new signal based triangular SE is introduced. In Section 3, a framework for fault diagnosis methodology is proposed. The fault diagnosis results and discussion are included in Section 4 . Section 5 presents the conclusions reached after the undertaken research.

\section{Mathematical Morphology and Correlation Analysis}

2.1. Basic Mathematical Morphological Operations. MM which originally aimed at quantifying the geometrical structure of image objects was initiated mainly by Maragos and Schafer [15] and Matheron [16] due to its robustness in both retaining image details and filtering noise. Its mathematical origins root in set theory, lattice algebra, convex analysis, and integral and stochastic geometry. It has rich theoretical framework and higher algorithmic efficiency and is easy to implement on special hardware. MM, nowadays, offers many theoretical and algorithmic tools, inspiring new directions in many research areas from the fields of signal processing, image processing, machine vision, and pattern recognition. As MM has been shown to successfully extract impulsive components from the original signal with background noise, it is adopted as a feature extractor in this paper to extract fault features from vibration signals of rolling element bearing.

The key idea of MM signal processing is to modify the shape of a signal by transformation through interaction with a SE in order to extract the feature information. The signal and SE are both treated as sets in morphological processing; the latter usually has a simpler shape and is more compact than the former [19]. The mathematical calculation involved in MM includes only addition, subtraction, maximum, and minimum operations without any multiplication or division. Upon interaction with complex shapes of signal, MM operations are capable of decomposing a signal into meaningful 
components and separating them from the background, as well as preserving the main shape characteristics [26]. The basic operators of MM include dilation, erosion, opening, and closing; they are defined as follows [24].

Let $f(n)$ represent a one-dimensional (1D) signal which is a discrete function over a domain $F=(0,1,2, \ldots, N-1)$. And let $g(m)$ denote a SE which is a discrete function over a domain $G=(0,1,2, \ldots, M-1)$. Two basic morphological operations, the erosion and dilation of $f$ by $g$, can be, respectively, defined as

$$
\begin{array}{r}
(f \Theta g)(n)=\min [f(n+m)-g(m)], \\
m \in 0,1,2, \ldots, M-1, \\
(f \oplus g)(n)=\max [f(n-m)+g(m)], \\
m \in 0,1,2, \ldots, M-1,
\end{array}
$$

where $\Theta$ denotes the operation of erosion and $\oplus$ denotes the operation of dilation.

Based on the erosion and dilation operations, the other two morphological operators, the opening and the closing of $f$ by $g$, can be further defined as

$$
\begin{aligned}
& (f \circ g)(n)=(f \Theta g \oplus g)(n), \\
& (f \circ g)(n)=(f \oplus g \Theta g)(n),
\end{aligned}
$$

where $\circ$ stands for the opening operator and $\bullet$ for the closing operator.

The above four operators can all be used in feature extraction of a signal, while other operations are chosen appropriately to extract different components of a signal. The dilation can broaden the width of positive peaks and reduce the width of negative peaks while the erosion of a signal gives and produces inverse effect. The opening and closing operation can remove positive and negative impulses while preserving other information. The properties of the four morphological operations for impulse features are listed in Table 1. Since the closing operation is proper for extraction of positive impulse, it is adopted in this paper for the signal processing of bearing signals.

2.2. The Signal Based Triangular SE. The performance of a morphological technique depends not only on the morphological operation, but also on the geometry of the SE employed. The design of a SE should depend on the signal waveform in MM analysis in order to extract feature information from the signal. The shape, length, and height are three parameters which should be determined in the construction of a SE. As discussed above, the triangular SE with the length scale of 3 is adopted in this paper as it is similar to every three adjacent points in a signal. In order to simplify the specification of a triangular SE to improve computational efficiency, the SEs used here are just isosceles triangular SEs.

After determining the shape and length of a SE, a proper height also needs to be determined. The height $(h)$ of a SE is usually decided according to prior experience [24]. However, the prior experience and knowledge are not always
TABLE 1: Properties of MM operations to impulse features.

\begin{tabular}{lcc}
\hline Morphological operation & Negative impulse & Positive impulse \\
\hline Erosion & Smoothing & Reducing \\
Dilation & Reducing & Smoothing \\
Opening & Preserving & Reducing \\
Closing & Reducing & Preserving \\
\hline
\end{tabular}

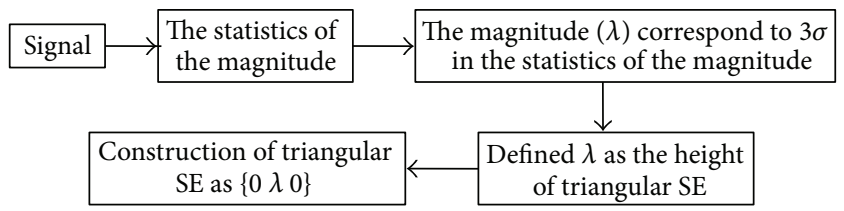

FIGURE 1: The construction procedure of triangular SE.

available, especially for new signals. Taking that into account, it may be a more feasible option if the height of a SE can be determined by considering every original unique signal. Assuming that the signal obeys the principle of $3 \sigma$ in the statistics of the magnitude, then one can consider that the data magnitudes beyond the range of $3 \sigma$ of a signal are mostly contributed by noise. Therefore, this paper defines the height of a triangular SE as the highest magnitude within the $3 \sigma$ of an analyzed signal. Using variable $\lambda$ representing this value, then the triangular SE can be described as $\{0 \lambda 0\}$. The detailed procedure of triangular SE construction is shown in Figure 1.

To illustrate the performance of triangular SE with different heights in signal processing, an example based on the morphological closing operation is given. Vibration signal $x$ is obtained from working bearing with an outer race fault. The heights of SEs are selected as $0.01,0.7$ (1 to 5 percent of the height in original signal [28]), 2.3 (calculated by the proposed procedure given above), and 10. The SE of $\left\{\begin{array}{lll}0 & 0.01 & 0\end{array}\right\}$ is like flat SE, while the SE of $\left\{\begin{array}{lll}0 & 10 & 0\end{array}\right\}$ is like an impulse. The other two SEs of $\left\{\begin{array}{lll}0 & 0.7 & 0\end{array}\right\}$ and $\left\{\begin{array}{lll}0 & 2.3 & 0\end{array}\right\}$ are obtained from signal. Processing results after the morphological closing operation with the four SEs are shown in Figure 2. The right parts in Figure 2 are local detail of the selected parts on the left figure. The black line and gray line represent the original signal $x$ and the processed signal, respectively. It can be demonstrated from Figures 2(a), 2(b), and 2(d) that the triangular SEs with small height will result in the loss of many negative peaks, which may contain feature information, while the triangular SEs with large height have no feature extraction ability. More feature components can be extracted by the proposed SE, as shown in Figure 2(c).

2.3. Correlation Analysis. Correlation analysis (CA) is a helpful tool in calculating the similarity of two sets of data of the same size. It was applied to evaluate the effectiveness of bearing fault diagnosis methods by Sun et al. [29] and Chen et al. [30]. The correlation degree of two data sets is often described by correlation coefficients which range in value from -1 to +1 . A value approaching +1 represents a perfect positive correlation between data while a value close to -1 represents a negative correlation. If no linear relationship 

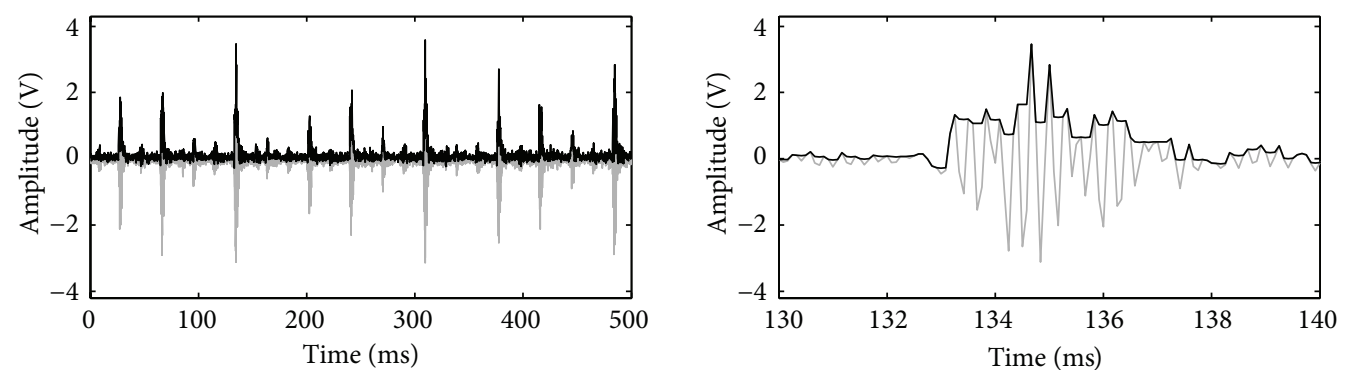

(a)
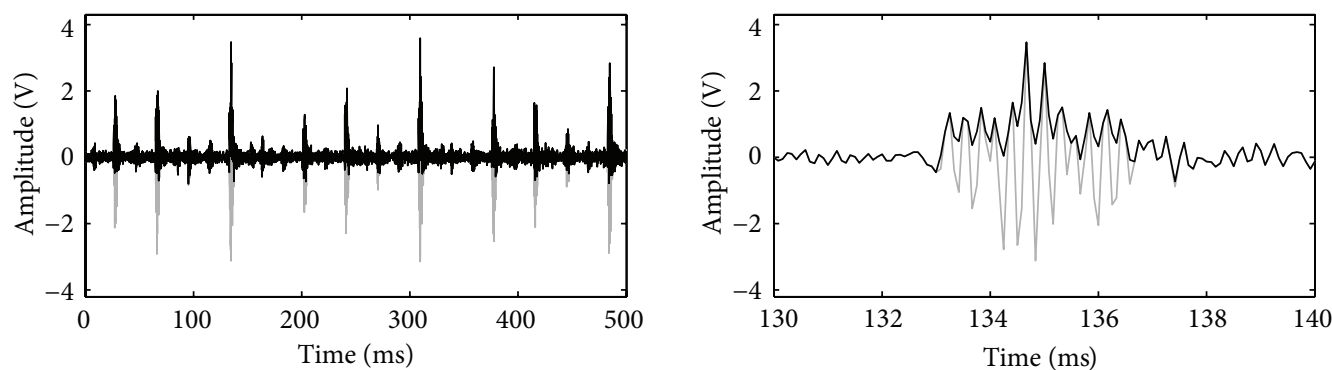

(b)
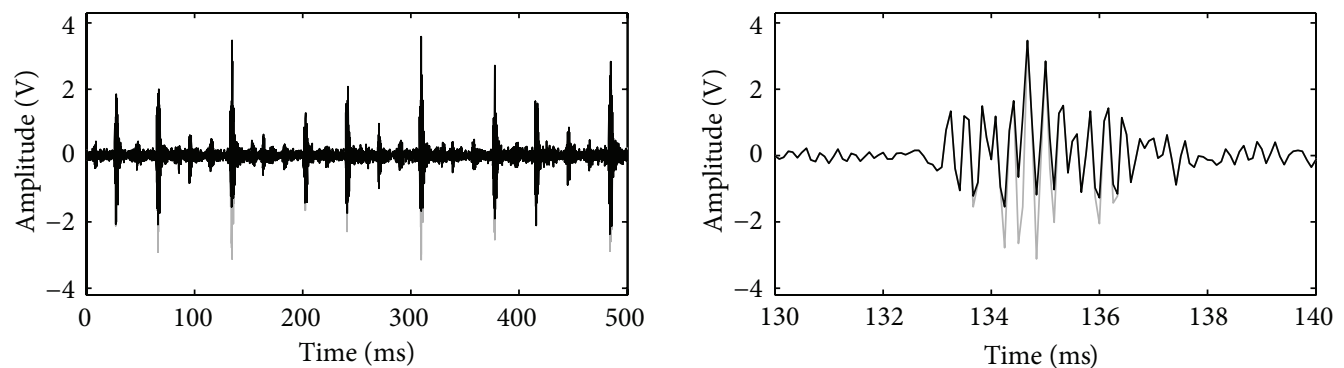

(c)
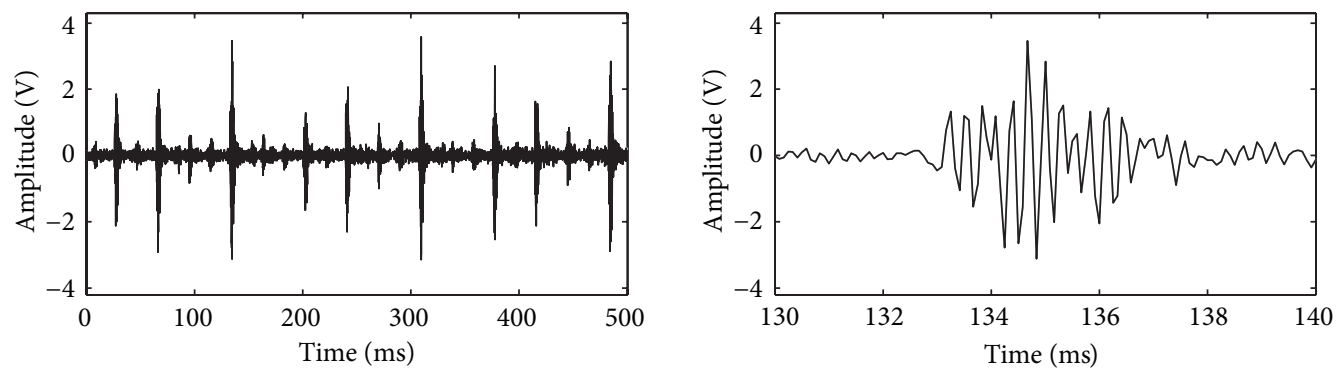

(d)

Figure 2: Signal after closing operation by triangular SEs with different heights: (a) $h=0.01$, (b) $h=0.7,(\mathrm{c}) h=2.3$, and (d) $h=10$.

exists between the two data sets, the correlation coefficient will be 0 .

To define the correlation coefficient [29], first consider the sum of squared values $s s_{x x}, s s_{x y}$, and $s s_{y y}$ of a set of $n$ data points $\left(x_{i}, y_{i}\right)$ about their respective means:

$$
\begin{gathered}
s s_{x x}=\sum\left(x_{i}-\bar{x}\right)^{2}, \\
s s_{y y}=\sum\left(y_{i}-\bar{y}\right)^{2}, \\
s s_{x y}=\sum\left(x_{i}-\bar{x}\right)\left(y_{i}-\bar{y}\right) .
\end{gathered}
$$

These quantities are simply nonnormalized forms of the variances and covariance of $X$ and $Y$ given by

$$
\begin{gathered}
s s_{x x}=\operatorname{Var}(X), \\
s s_{y y}=\operatorname{Var}(Y), \\
s s_{x y}=\operatorname{Cov}(X, Y) .
\end{gathered}
$$

The correlation coefficient $r$ is then defined by

$$
r=\sqrt{\frac{s s_{x y}^{2}}{s s_{x x} s s_{y y}}}=\frac{\operatorname{Cov}(X, Y)}{\sqrt{\operatorname{Var}(X) \operatorname{Var}(Y)}} .
$$




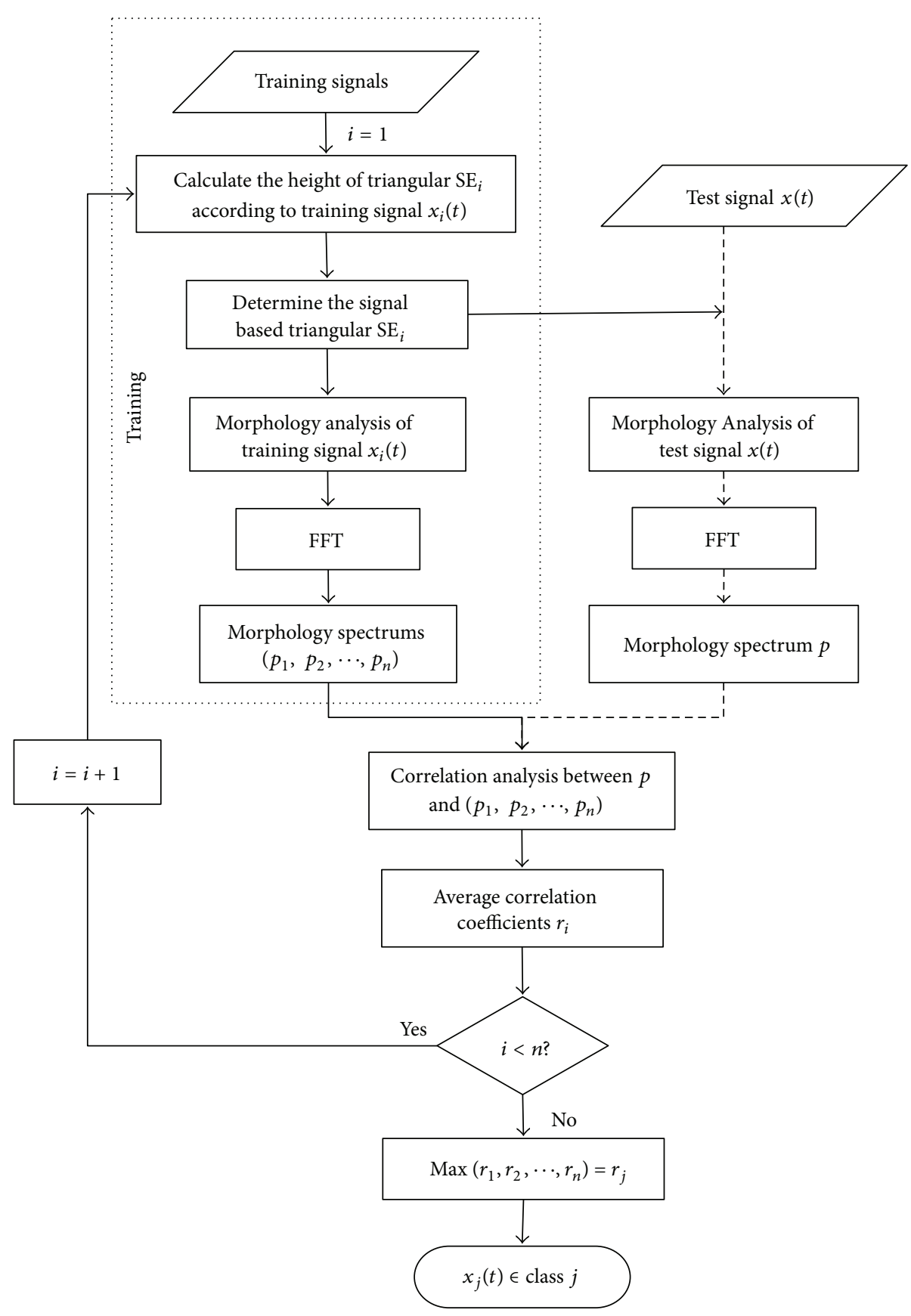

FIGURE 3: The fault diagnosis framework.

\section{Methodology}

In bearing system, faults usually occur in inner race, outer race, or rolling elements. When a running roller passes over the surfaces with defects, impulses will be generated. Those impulses are almost periodical and their characteristics depend on the location of defect. Thus, defect information is contained in the spacing of impulses rather than in their frequency content [31]. So if the feature of each fault can be extracted successfully, a proper classification of bearing faults can be achieved [29].
The proposed diagnosis method can be conducted in four steps as shown in Figure 3: the construction of a triangular $\mathrm{SE}$, feature extraction based on MM analysis, and fast Fourier transform (FFT) to get the morphology spectrum and the identification of bearing fault.

The detail of the proposed method can be described as follows.

(1) Training signals are divided into several data sets $\left\{x_{i}\right\}(i=1,2, \ldots, n)$ according to their failure conditions. The signal based triangular SEs are also 


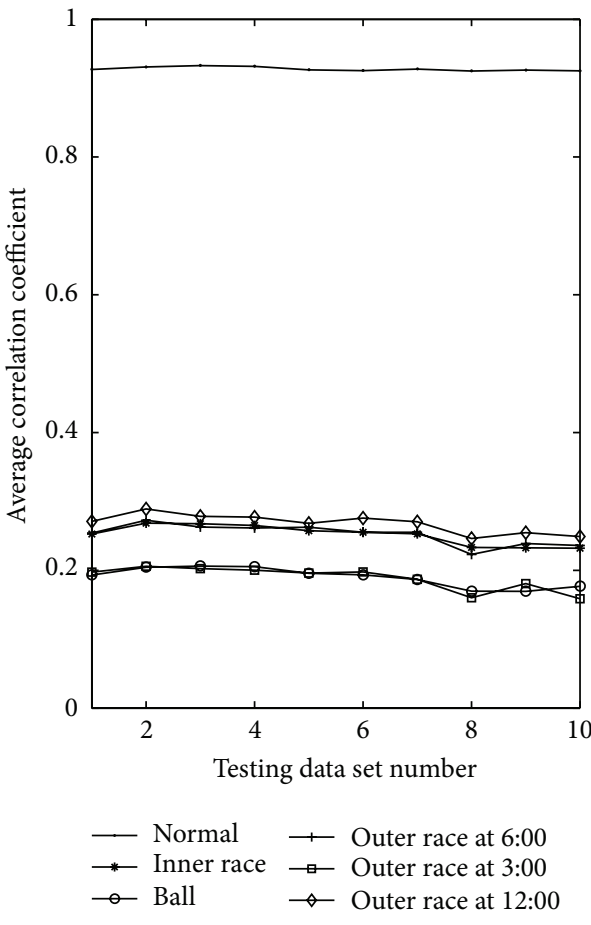

(a)

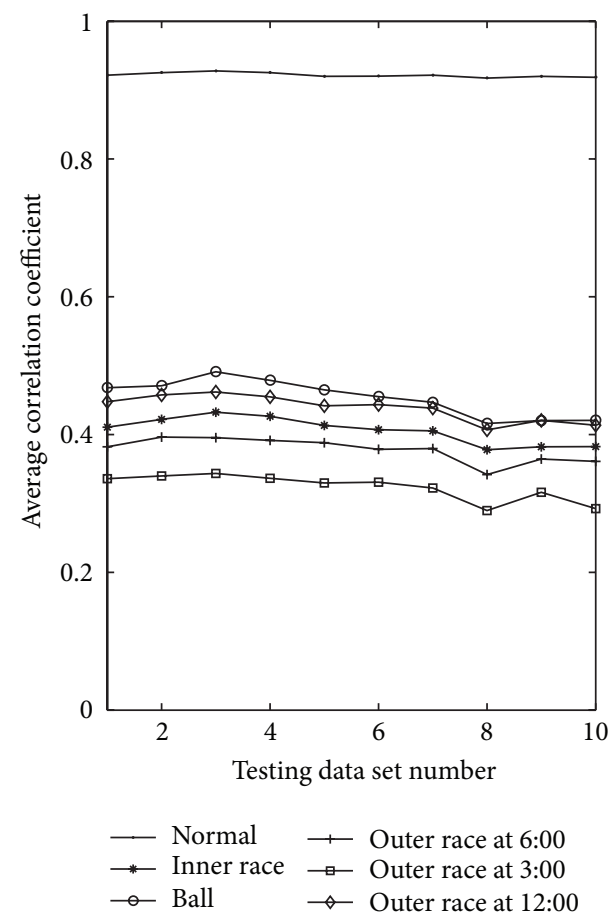

(b)

FIGURE 4: Detection results of bearing under normal condition according to morphology spectrum correlation analysis: (a) signal based triangular SE; (b) flat SE.

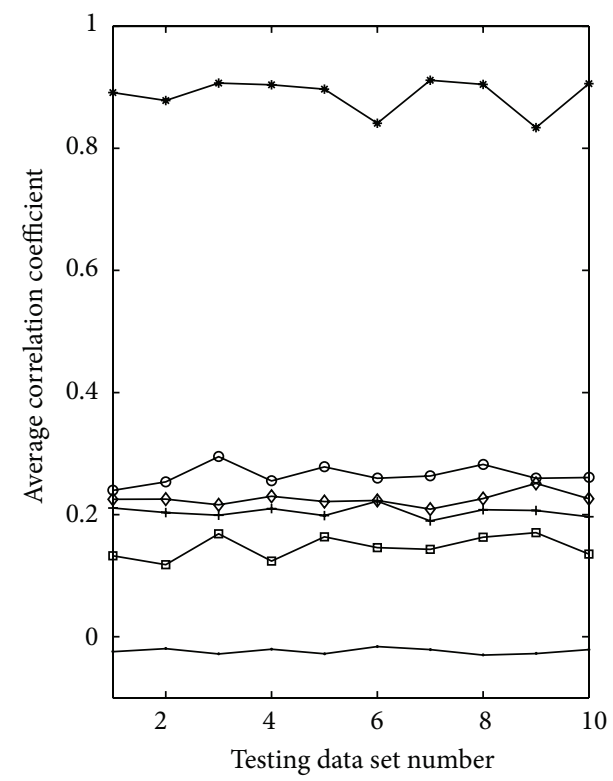

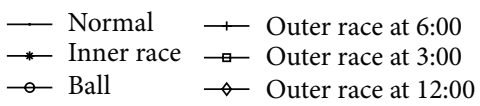

(a)

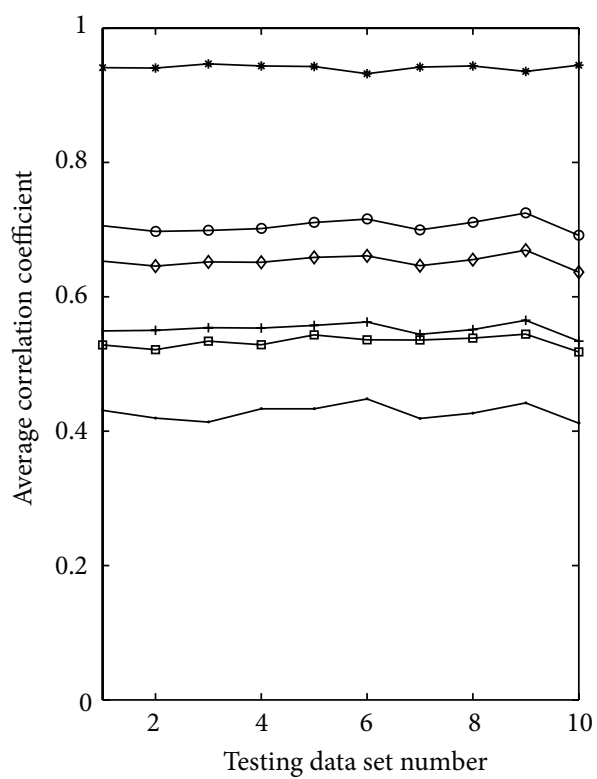

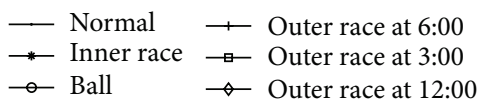

(b)

FIgURE 5: Detection results of data set A with an inner race fault according to morphology spectrum correlation analysis: (a) signal based triangular SE; (b) flat SE. 


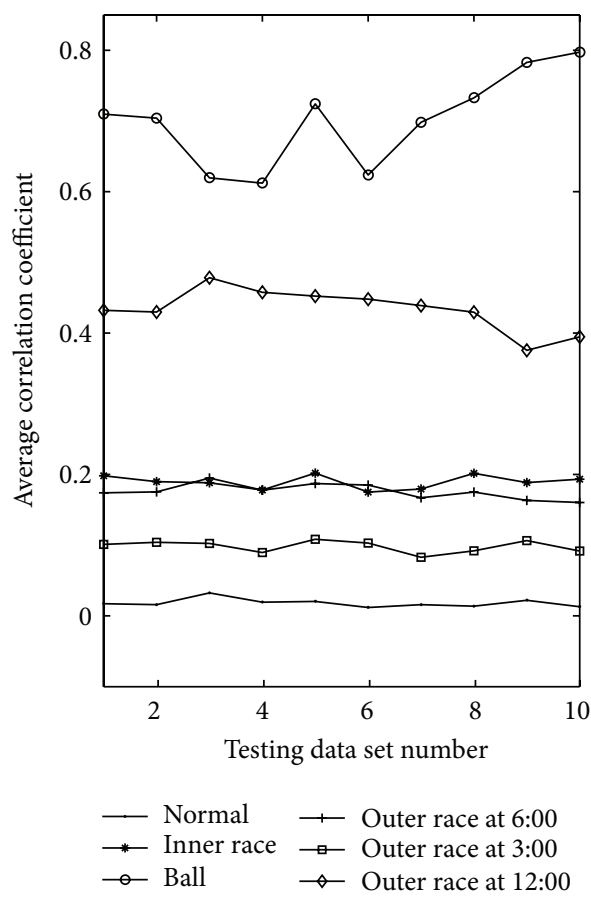

(a)

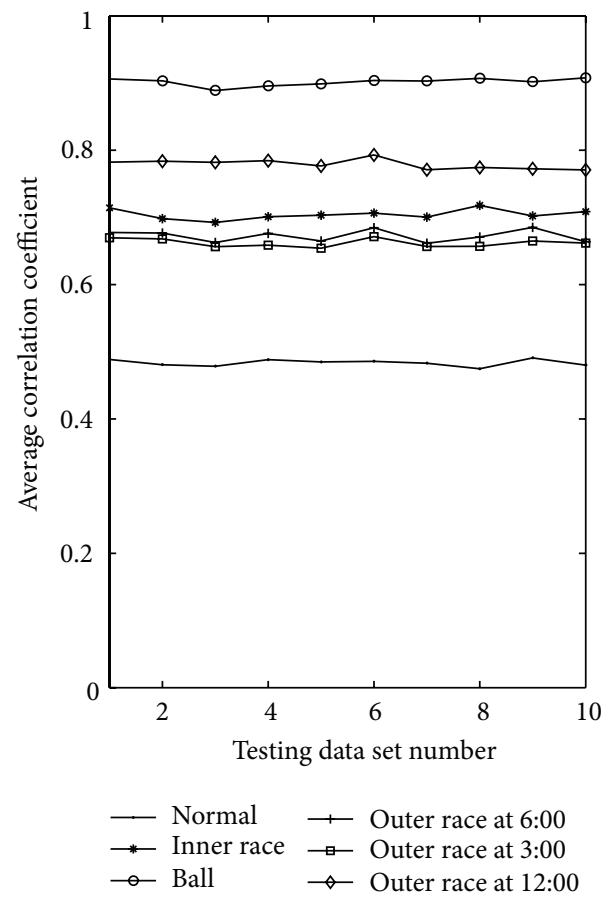

(b)

FIGURE 6: Detection results of data set A with a ball fault according to morphology spectrum correlation analysis: (a) signal based triangular SE; (b) flat SE.
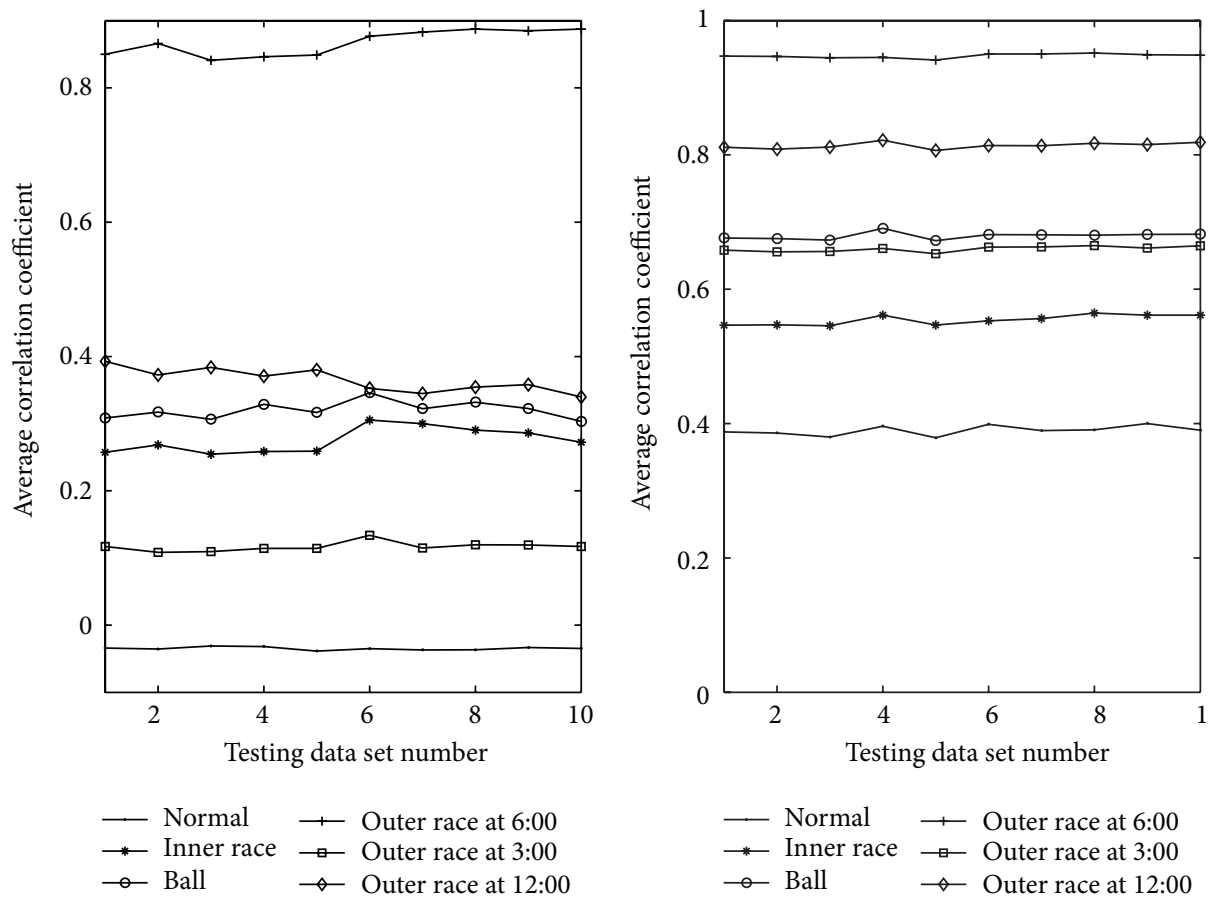

(a)

(b)

FIGURE 7: Detection results of data set A with an outer fault located at 6:00 according to morphology spectrum correlation analysis: (a) signal based triangular SE; (b) flat SE. 

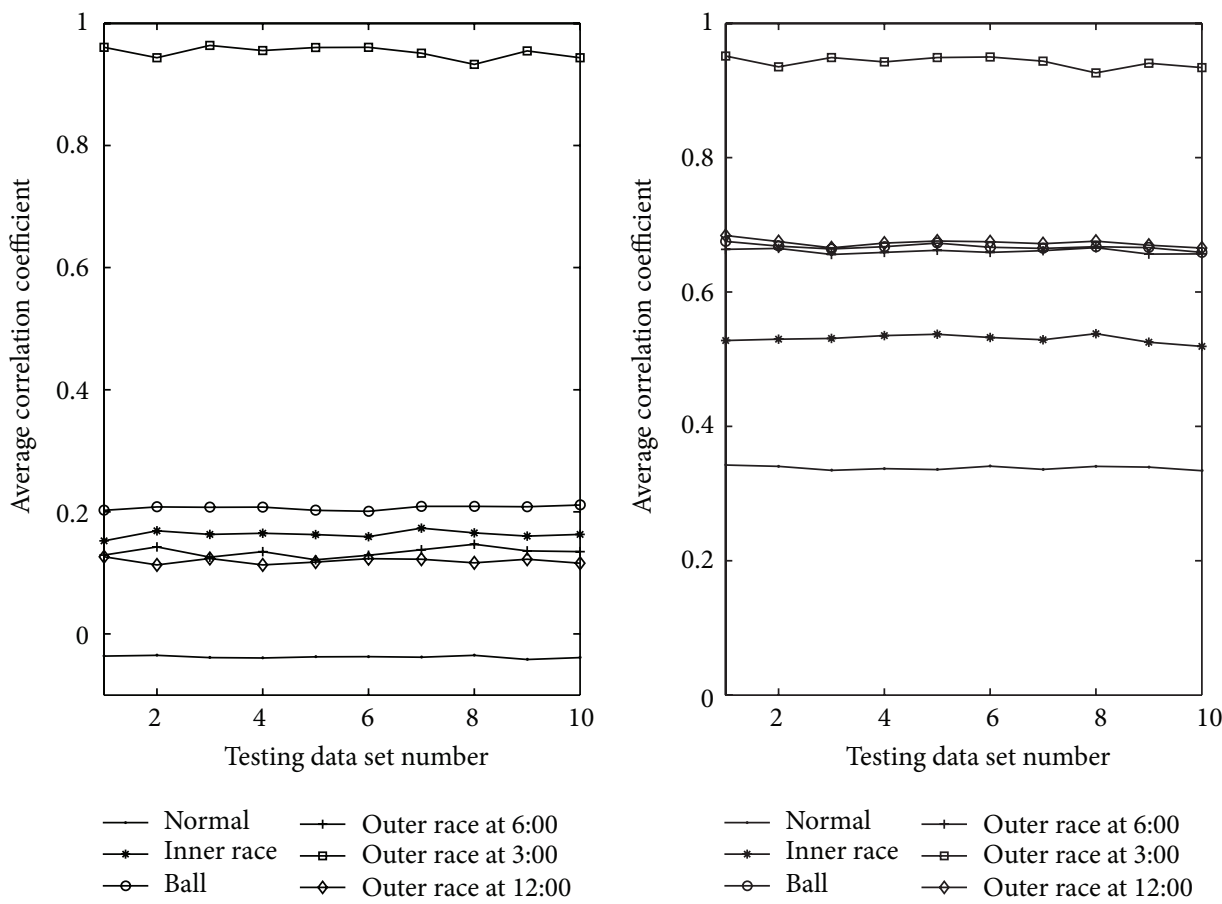

(b)

FIGURE 8: Detection results of data set A with an outer fault located at 3:00 according to morphology spectrum correlation analysis: (a) signal based triangular SE; (b) flat SE.
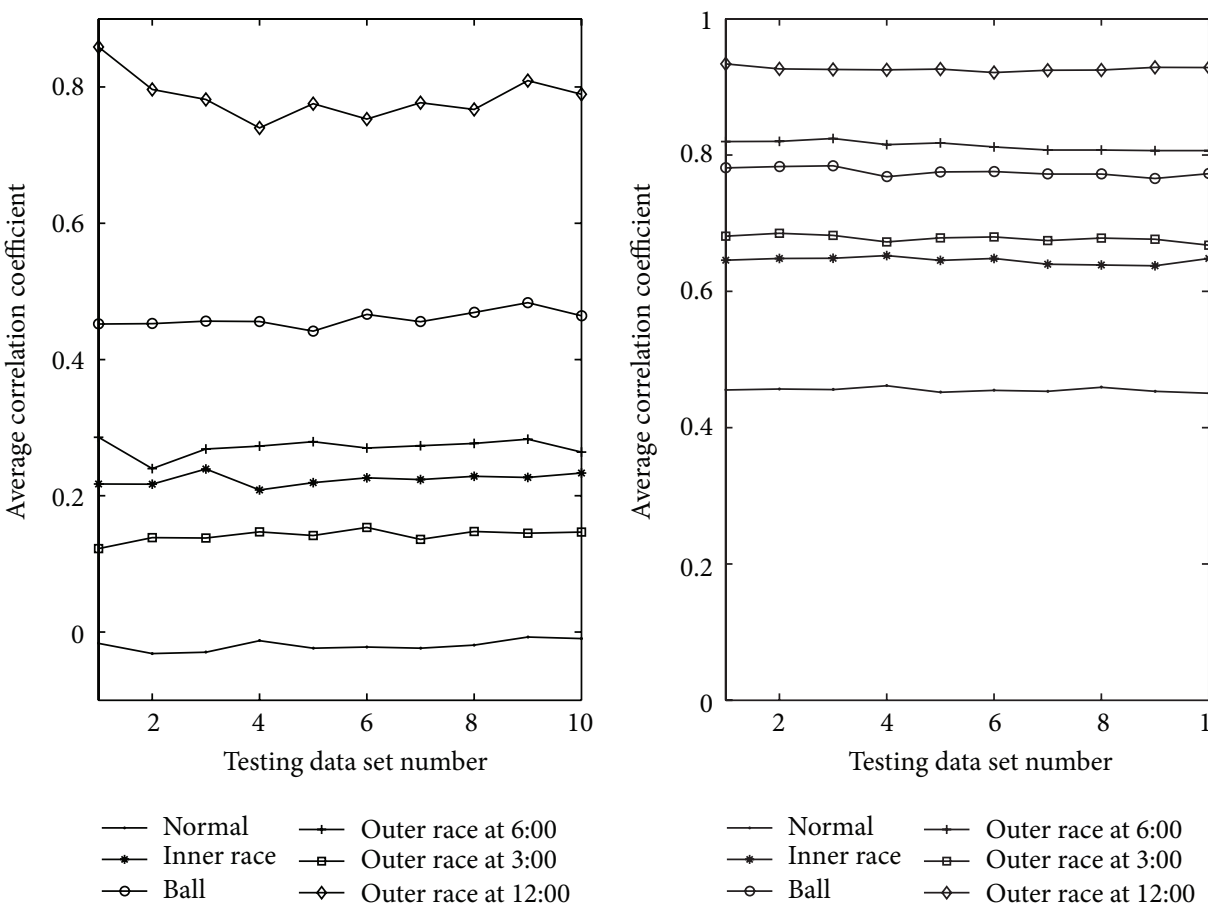

(a)

(b)

FIGURE 9: Detection results of data set A with an outer fault located at 12:00 according to morphology spectrum correlation analysis: (a) signal based triangular SE; (b) flat SE. 


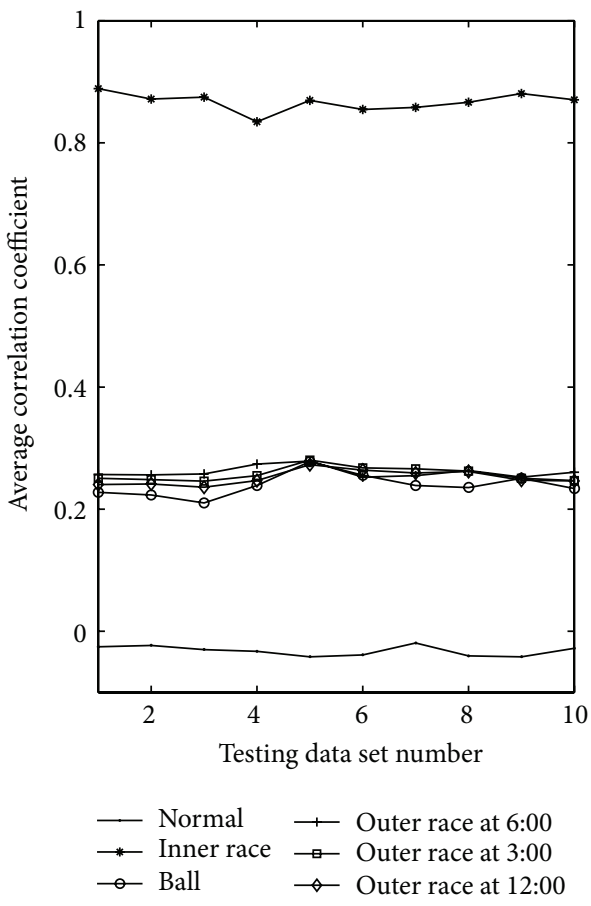

(a)

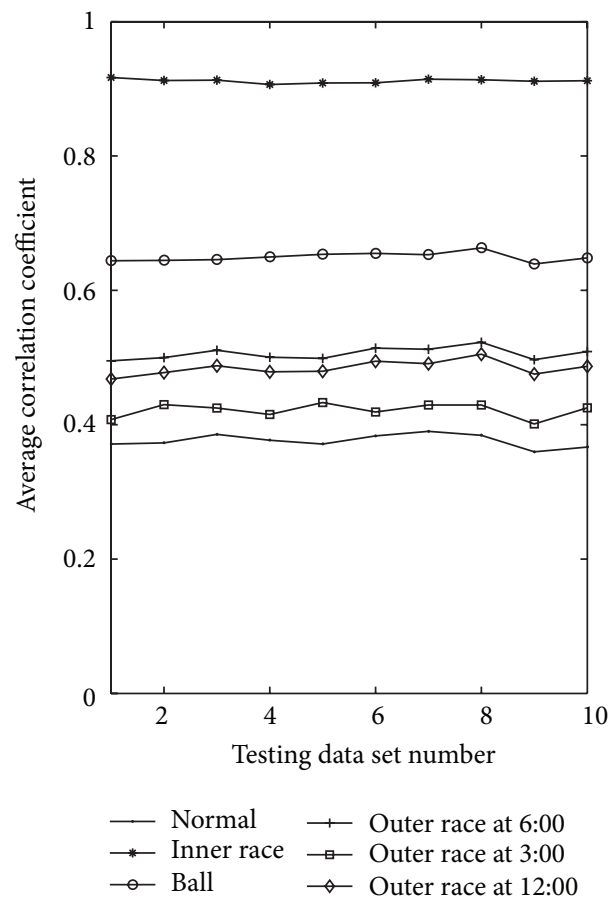

(b)

FIGURE 10: Detection results of data set B with an inner race fault according to morphology spectrum correlation analysis: (a) signal based triangular SE; (b) flat SE.

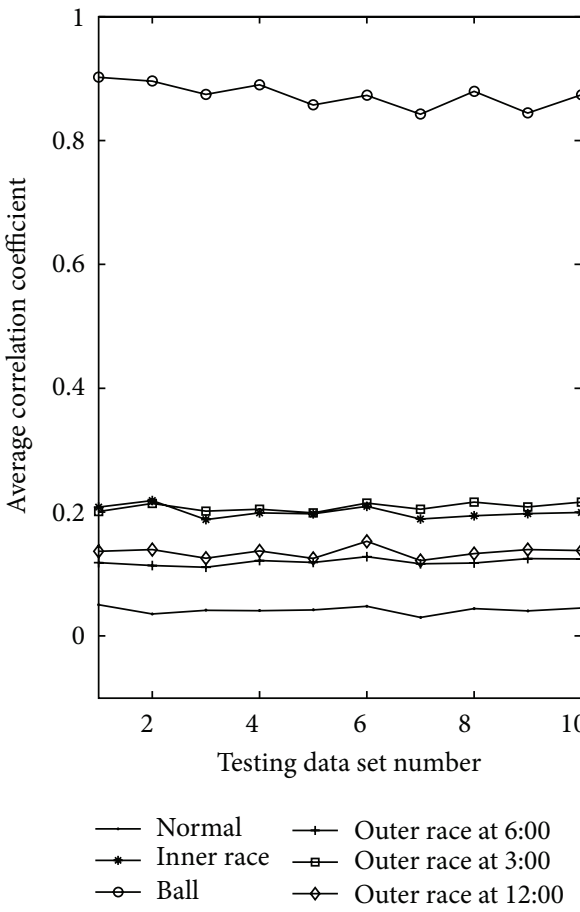

(a)

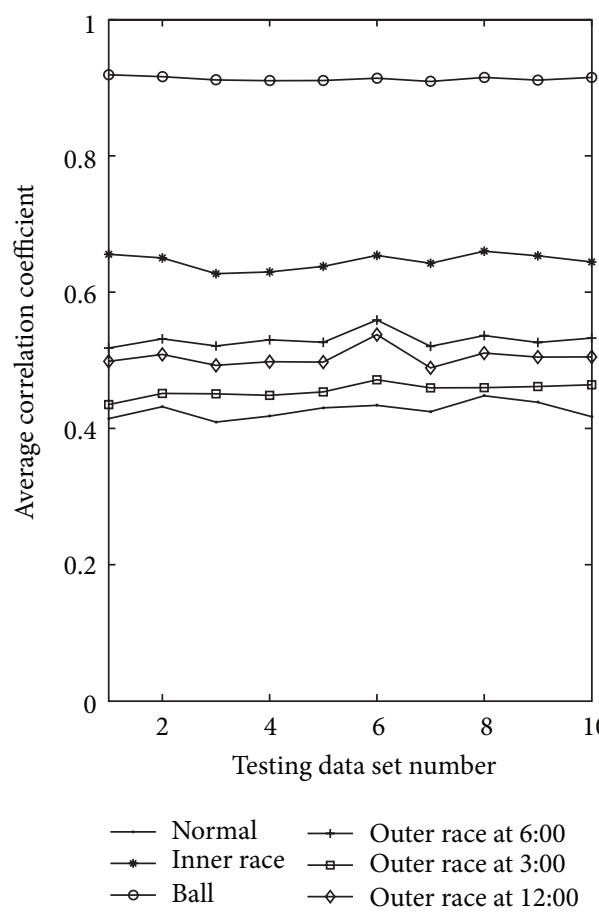

(b)

FIGURE 11: Detection results of data set B with a ball fault according to morphology spectrum correlation analysis: (a) signal based triangular SE; (b) flat SE. 


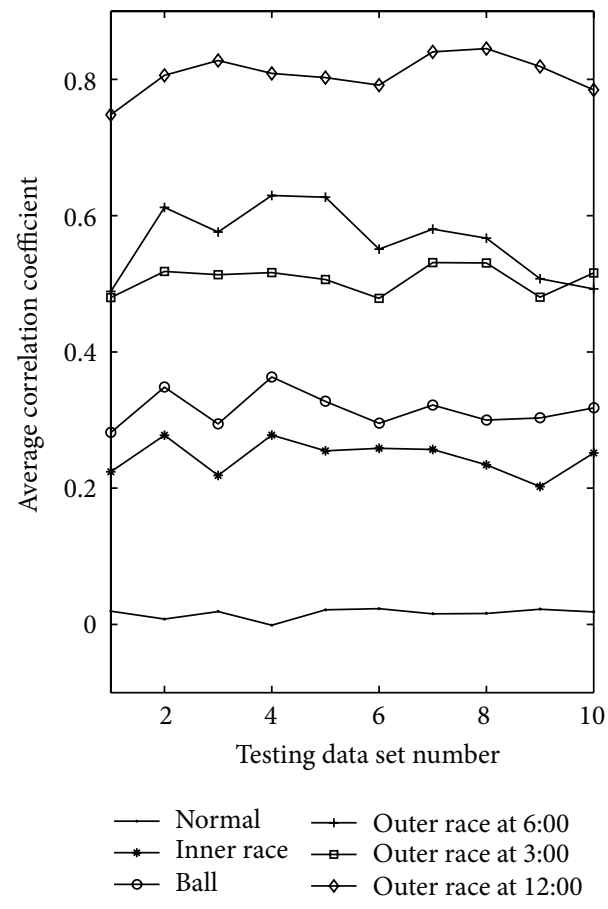

(a)

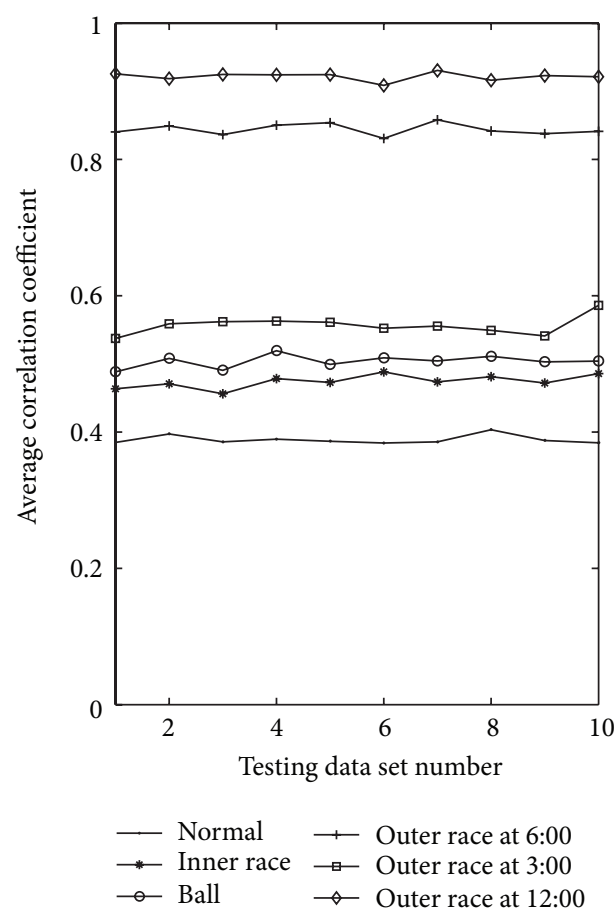

(b)

FIGURE 12: Detection results of data set B with an outer fault located at 12:00 according to morphology spectrum correlation analysis: (a) signal based triangular SE; (b) flat SE.

TABle 2: Details of data sets A, B, and C.

\begin{tabular}{|c|c|c|c|c|}
\hline Data set & $\begin{array}{l}\text { The number of } \\
\text { training samples }\end{array}$ & $\begin{array}{c}\text { The number of } \\
\text { test samples }\end{array}$ & $\begin{array}{c}\text { Defect size } \\
\text { (inches) }\end{array}$ & Operating condition \\
\hline \multirow{6}{*}{ A } & 10 & 10 & 0 & Normal \\
\hline & 10 & 10 & 0.007 & Inner race fault \\
\hline & 10 & 10 & 0.007 & Ball fault \\
\hline & 10 & 10 & 0.007 & Outer race fault (at 6:00) \\
\hline & 10 & 10 & 0.007 & Outer race fault (at 3:00) \\
\hline & 10 & 10 & 0.007 & Outer race fault (at 12:00) \\
\hline \multirow{6}{*}{ B } & 10 & 10 & 0 & Normal \\
\hline & 10 & 10 & 0.021 & Inner race fault \\
\hline & 10 & 10 & 0.021 & Ball fault \\
\hline & 10 & 10 & 0.021 & Outer race fault (at 6:00) \\
\hline & 10 & 10 & 0.021 & Outer race fault (at 3:00) \\
\hline & 10 & 10 & 0.021 & Outer race fault (at 12:00) \\
\hline \multirow{11}{*}{$\mathrm{C}$} & 10 & 10 & 0 & Normal \\
\hline & 10 & 10 & 0.007 & Inner race fault \\
\hline & 10 & 10 & 0.007 & Ball fault \\
\hline & 10 & 10 & 0.007 & Outer race fault (at 6:00) \\
\hline & 10 & 10 & 0.007 & Outer race fault (at 3:00) \\
\hline & 10 & 10 & 0.007 & Outer race fault (at 12:00) \\
\hline & 10 & 10 & 0.021 & Inner race fault \\
\hline & 10 & 10 & 0.021 & Ball fault \\
\hline & 10 & 10 & 0.021 & Outer race fault (at 6:00) \\
\hline & 10 & 10 & 0.021 & Outer race fault (at 3:00) \\
\hline & 10 & 10 & 0.021 & Outer race fault (at 12:00) \\
\hline
\end{tabular}




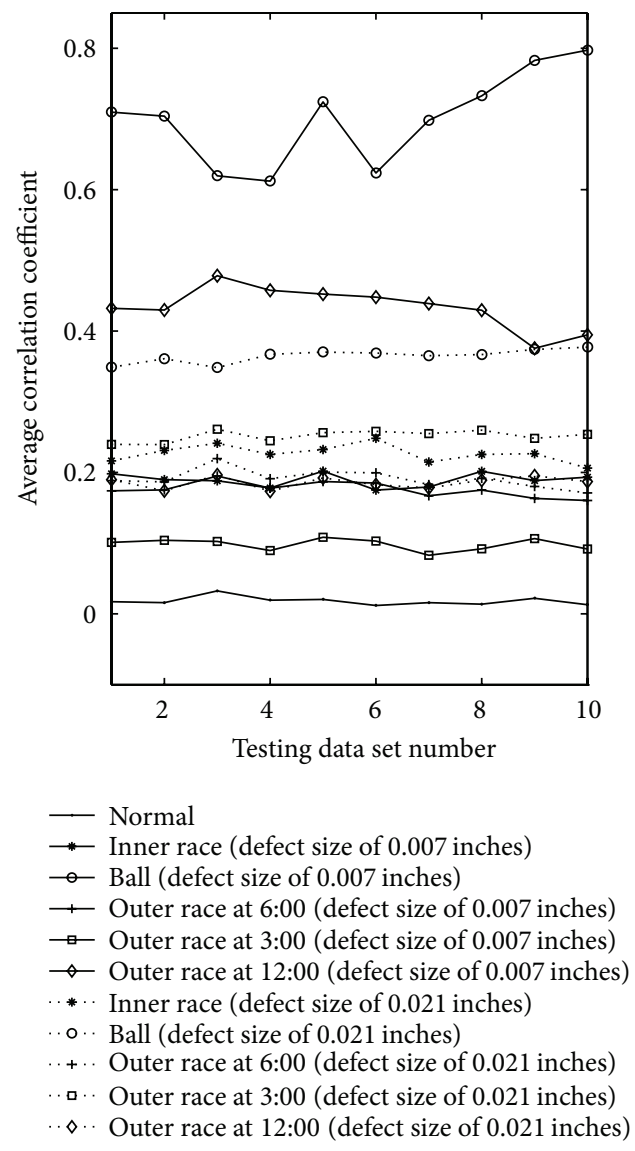

(a)

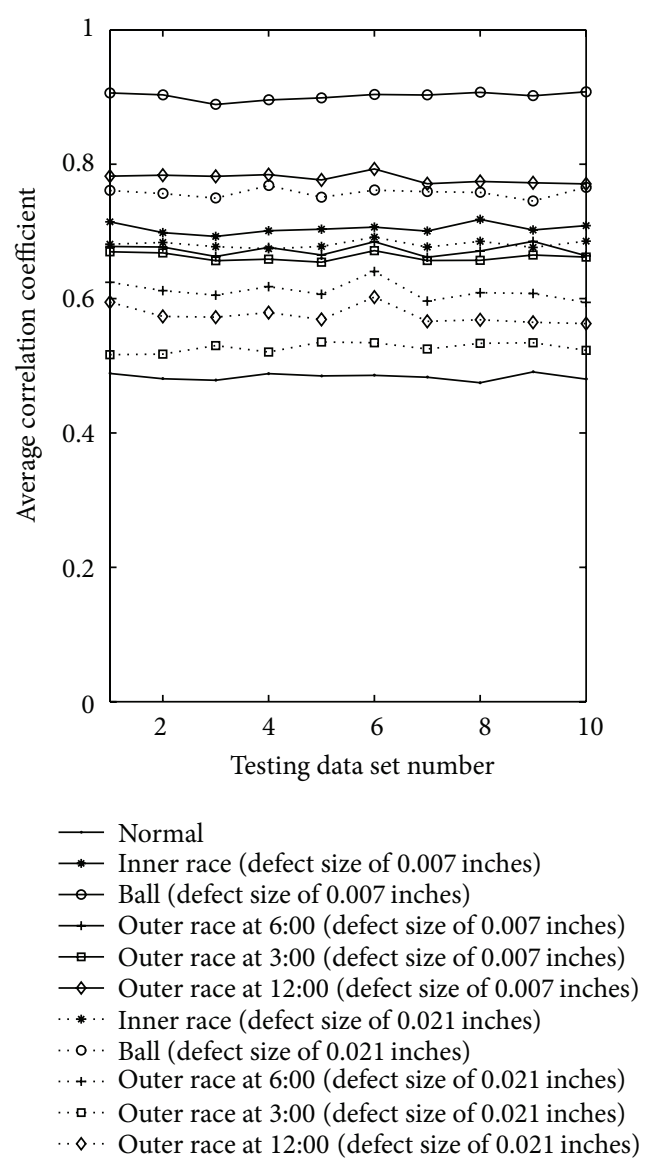

(b)

Figure 13: Detection results of ball fault with defect size of 0.007 inches according to morphology spectrum correlation analysis: (a) signal based triangular SE; (b) flat SE.

constructed in this step using the method given in Section 2.2.

(2) The closing operation and signal based triangular SE are applied to MM analysis for the signal feature extraction of training signals.

(3) The morphology spectrum sets $\left\{p_{i}\right\}(i=1,2, \ldots, n)$ of training signal can be obtained from processed signals by FFT.

(4) The test signal $x$ is analyzed following similar steps and its morphology spectrum $p$ is obtained. The correlation analysis on morphology spectrum is then employed to calculate the average correlation coefficient $r_{i}(i=1,2, \ldots, n)$ between $p$ and sets $\left\{p_{i}\right\}$ $(i=1,2, \ldots, n)$. Here the morphology spectrum correlation coefficient of two spectrums can be expressed as

$$
r=\frac{\operatorname{Cov}\left(p_{1}, p_{2}\right)}{\sqrt{\operatorname{Var}\left(p_{1}\right) \operatorname{Var}\left(p_{2}\right)}}
$$

where $r_{j}$ is assumed to be the maximum one in $r_{1}$ to $r_{n}$. The test signal $x$ is considered in the same operating condition with corresponding training signal set $\left\{x_{j}\right\}$.

\section{Fault Diagnosis of Rolling Element Bearings}

4.1. Experimental System and Data Description. The vibration signals used in this paper were acquired from the Bearing Data Center website of CWRU [27]. The CWRU bearing data has been used in many previous studies [11, 24, 32] and has become a benchmark signal for bearing fault diagnosis.

Vibration data used in this study was collected under $3 \mathrm{hp}$ load using accelerometers, which were attached to the housing with magnetic bases. The sampling frequency was $12,000 \mathrm{~Hz}$ per channel. The bearing used in the experiments was a type SKF 6205, deep groove ball bearing. Single point faults were introduced to the test bearings using electrodischarge machining with fault diameters of 0.007 inches and 0.021 inches, and the fault depth was 0.011 inches. The bearing data sets were obtained under normal, inner race fault, ball fault, and outer race fault conditions. As the outer raceway was stationary, the fault location relative to the load zone of the bearing had a direct impact on the vibration response. The outer race faults were located at 3 o'clock (3:00), 6 o'clock (6:00), and 12 o'clock (12:00), respectively. So the data was collected under six operating conditions. 

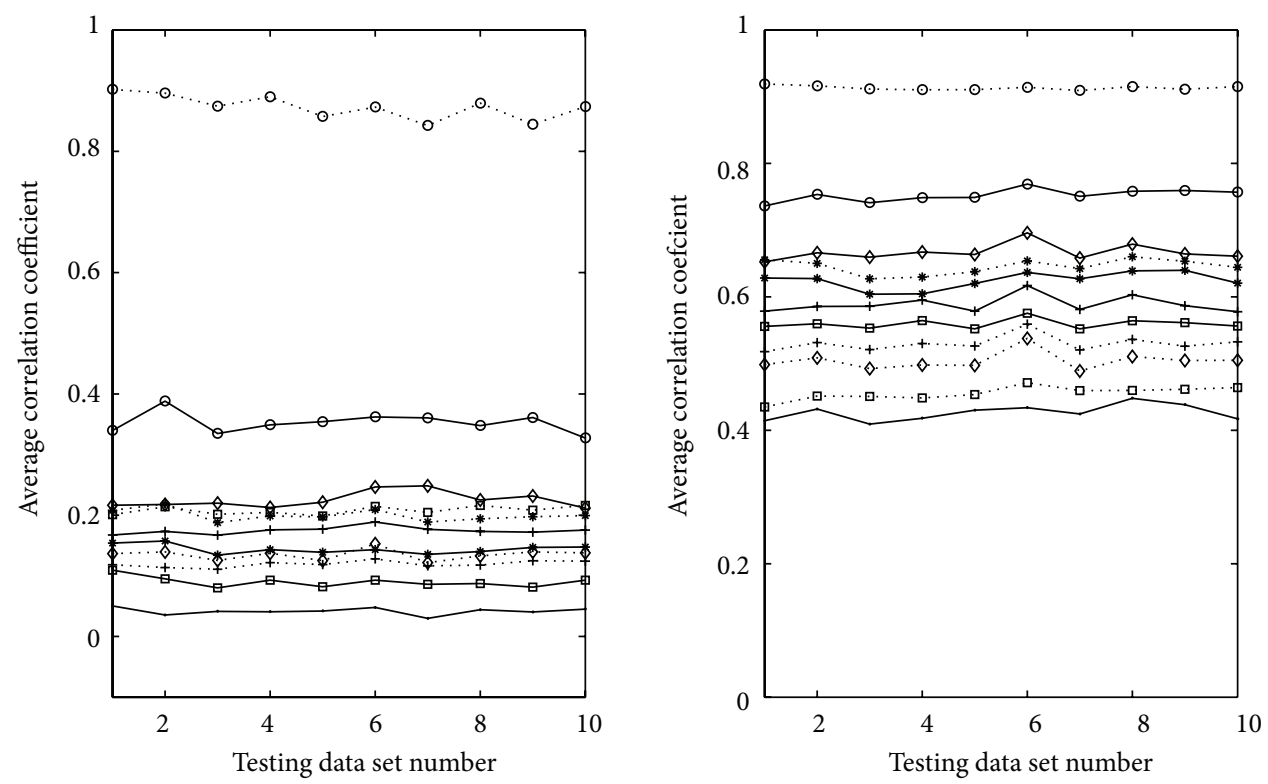

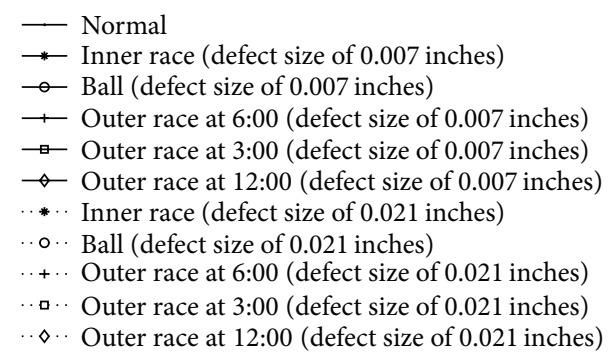

(a)

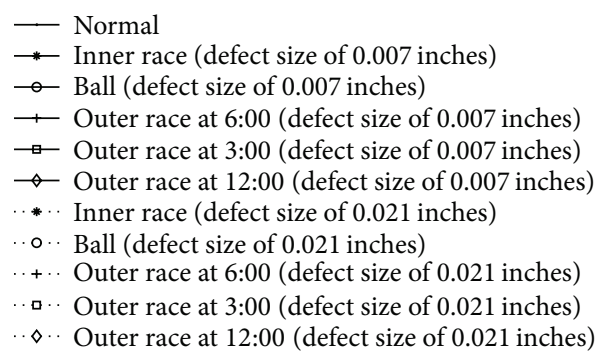

(b)

FIGURE 14: Detection results of ball fault with defect size of 0.021 inches according to morphology spectrum correlation analysis: (a) signal based triangular SE; (b) flat SE.

As shown in Table 2, data used in this paper is divided into three data sets $(\mathrm{A}-\mathrm{C})$ in order to evaluate the performance of the proposed approach. Data set A consists of 120 samples under normal condition and other five kinds of fault conditions with defect size of 0.007 inches. Each operating condition includes 20 samples: ten samples for training and ten samples for test. Each sample consists of 6,000 data points. Hence the bearing fault diagnosis of data set $A$ is a six-class classification. Data set B is similar to data set A, except that the fault defect size is 0.021 inches. It is used for further validation of the classification ability in bearing detection under other defect sizes. Data set $\mathrm{C}$ is formed by a combination of data sets $\mathrm{A}$ and $\mathrm{B}$. It contains 220 samples which are divided into 110 training and 110 test patterns. The data set $\mathrm{C}$ is utilized to classify the severity of fault.

\subsection{Detection Results of Rolling Element Bearing}

4.2.1. Detection of Bearing under Normal Condition. As the data samples of bearing under normal condition in data sets $\mathrm{A}, \mathrm{B}$, and $\mathrm{C}$ are the same, the detection results of normal bearing are given only once. In order to demonstrate the feature extraction performance of proposed signal based triangular SE, the commonly used flat SE with the same length as the proposed SE is employed for a comparison. The detection results are given by correlation analysis on morphology spectrum with the proposed SE and flat SE given in Figures 4(a) and 4(b), respectively. There are 10 points marked on each line. Each point indicates the average morphology spectrum correlation coefficient between test data and training data samples. In Figure 4, the points in the top line represent the average morphology correlation coefficients between test data and normal training data. That means the correlation coefficients of the test data and normal training data samples are the largest. So the test data is classified as normal, which is consistent with the actual operation. The normal condition can be correctly detected by the two SEs, but the signal based triangular SE yields a slightly better performance at classification than flat SE.

4.2.2. Detection of Data Set $A$ with an Inner Race Fault. The fault diagnosis results of bearing with an inner race fault are given in Figure 5. It can be seen that the line of the average correlation coefficients between test data and training data samples under inner race fault is much higher than others. The test data is most relevant to training data samples with 

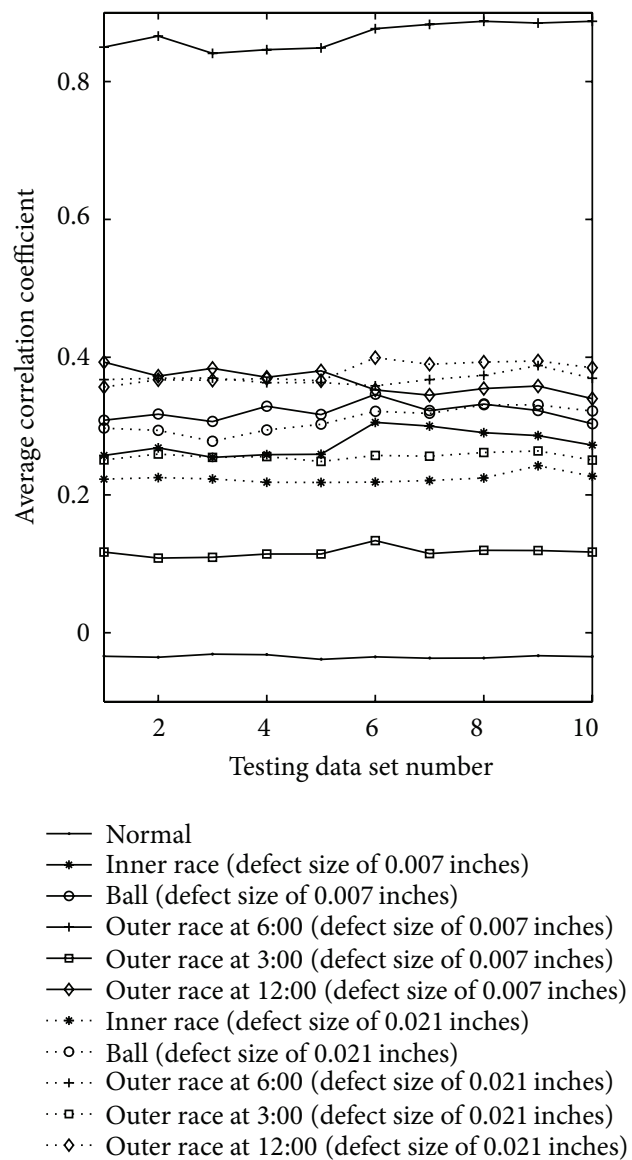

(a)

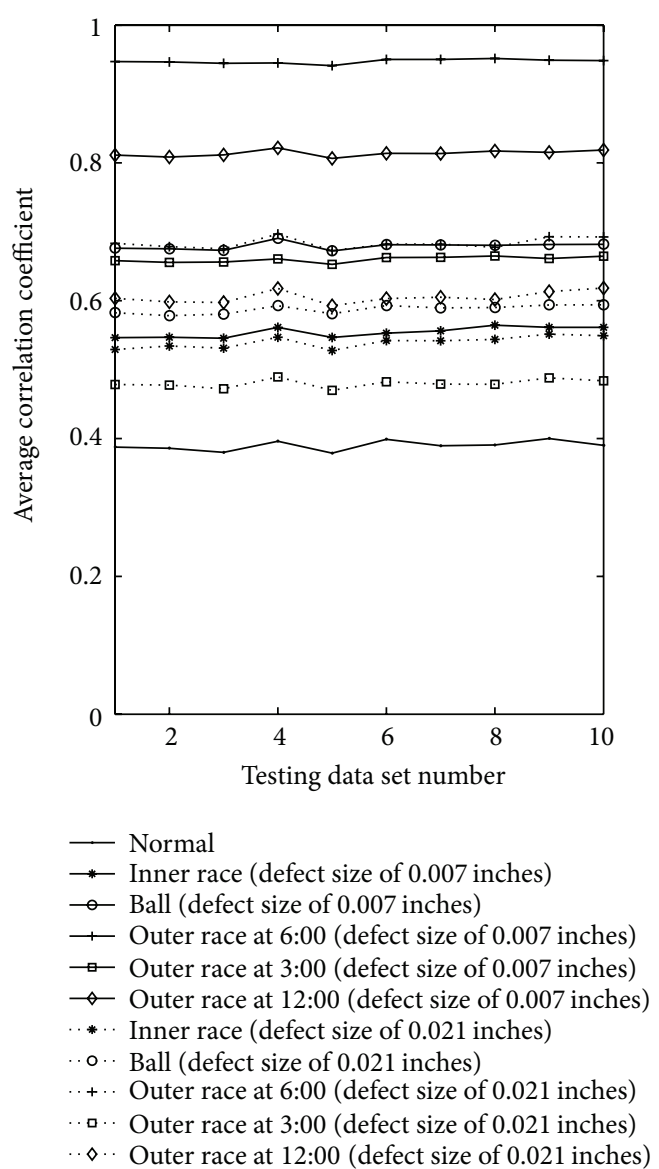

(b)

FIGURE 15: Detection of outer race fault at 6:00 with defect size of 0.007 inches according to morphology spectrum correlation analysis: (a) signal based triangular SE; (b) flat SE.

inner race fault. Hence the operating condition with inner race fault can be recognized clearly by both SEs. The proposed SE performs better than the flat SE in detection of inner race fault.

4.2.3. Detection of Data Set $A$ with a Ball Fault. The detection of bearing with a ball fault is usually difficult by using traditional envelope analysis because the features for a ball fault are very weak [33]. Figure 6 shows the detection results by the proposed method based on the two SEs. As seen in the figure, the morphology spectrum of test data is apparently relevant to that of the training data samples with ball fault. Both the SEs can give the right classification results; however, the result in Figure 6(a) is much clearer than that in Figure 6(b).

4.2.4. Detection of Data Set A with an Outer Race Fault. As the outer race faults have different impact on bearing vibration depending on the fault location, the rough location detection is very meaningful in machine security. Since each morphology spectrum of outer race fault with a certain defect position is unique, the morphology spectrum can be used for classification of the defect location in outer race. The detection results of outer race fault located at 6:00, 3:00, and 12:00 are shown in Figures 7 to 9, respectively. It can be demonstrated from Figures 7(a), 8(a), and 9(a) that the three faults can be correctly and clearly identified by the proposed method with signal based triangular SE. Although the results according to the proposed method with flat SE in Figures 7(b), 8(b), and 9(b) are all right, there are still certain rooms for the improvement of classification performance. Therefore, the signal based triangular SE performs better than flat SE in fault diagnosis at detecting the location of bearing with outer race fault.

4.2.5. Detection of Data Set B. Data set B is used to further test the effectiveness of the proposed method and SE in fault detection of bearing with defect size of 0.021 inches. The classification results of data set $B$ have been shown to be $100 \%$ correct. The detection results of data set B with an inner race fault, a ball fault, and an outer race fault located at 12:00 are shown in Figures 10 to 12 . As can be seen, the distance between the first line and the second line in Figures 10(a), 11(a), and 12(a) is much bigger than that in 


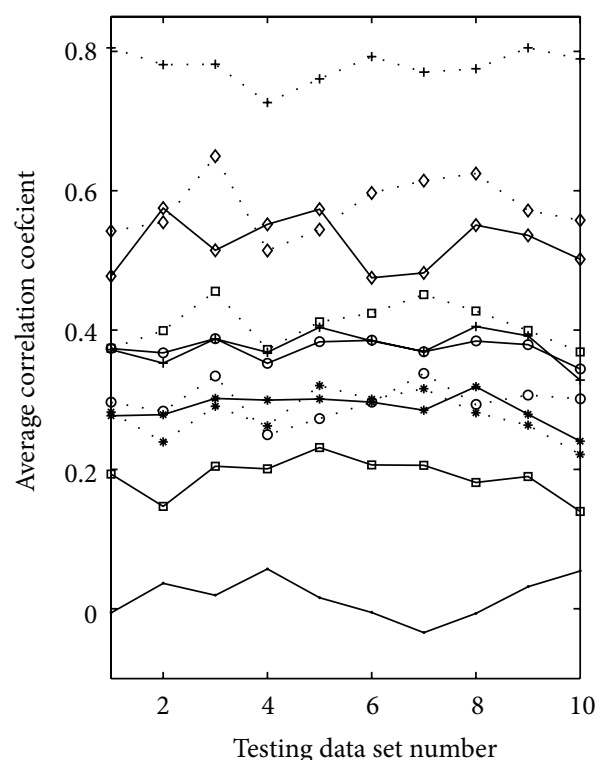

$\longrightarrow$ Normal
$\rightarrow$ Inner race (defect size of 0.007 inches)
$\rightarrow$ Ball (defect size of 0.007 inches)
$\rightarrow$ Outer race at $6: 00$ (defect size of 0.007 inches)
$\rightarrow-$ Outer race at 3:00 (defect size of 0.007 inches)
$\rightarrow-$ Outer race at 12:00 (defect size of 0.007 inches)
$\cdots * \cdots$ Inner race (defect size of 0.021 inches)
$\cdots \circ$ Ball (defect size of 0.021 inches)
$\cdots+\cdots$ Outer race at $6: 00$ (defect size of 0.021 inches)
$\cdots \bullet$ Outer race at 3:00 (defect size of 0.021 inches)
$\cdots \diamond \cdots$ Outer race at 12:00 (defect size of 0.021 inches)

(a)
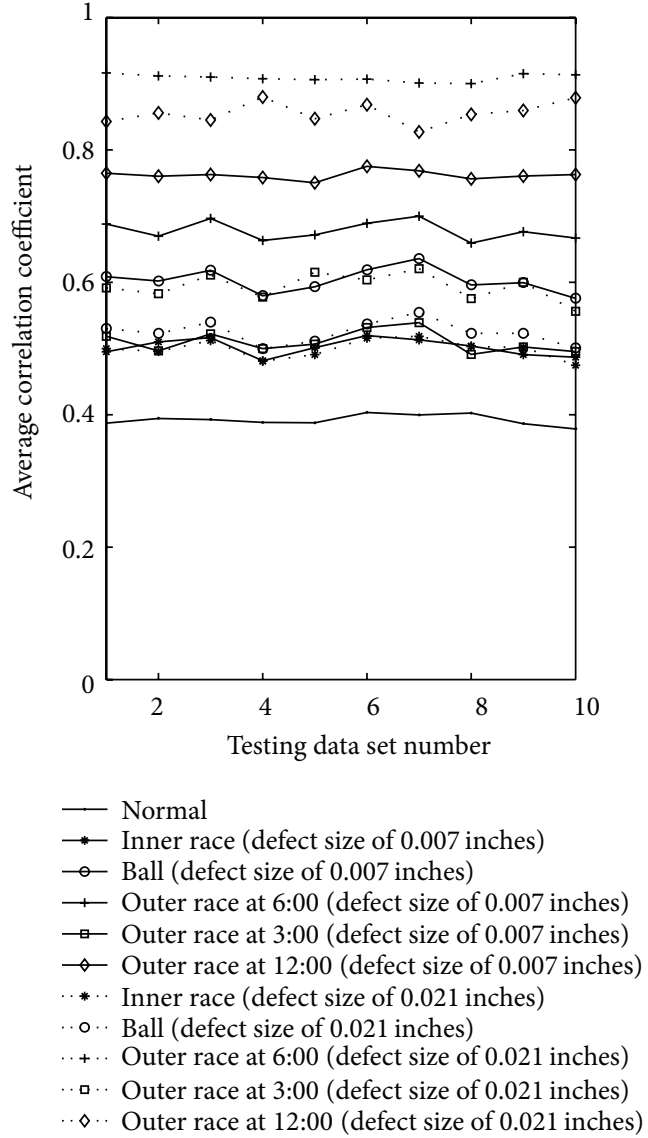

(b)

FIGURE 16: Detection of outer race fault at 6:00 with defect size of 0.021 inches according to morphology spectrum correlation analysis: (a) signal based triangular SE; (b) flat SE.

Figures 10(b), 11(b), and 12(b). In other words, the proposed method with the signal based triangular SE is more proper for fault diagnosis of bearing defect size of 0.021 inches. Figures 6(a) and 12(a) demonstrate that the ball fault can be identified more clearly as the defects get more serious by the proposed method. The fault diagnosis results of data set B also show that the proposed SE performs better than flat SE.

4.2.6. Detection of Data Set C. Data set C is adopted to verify the validity of the proposed approach with the signal based triangular SE in the classification of bearing faults with different defect severity. Some detection results of data set $\mathrm{C}$ are shown in Figures 13, 14, 15, and 16. The bold lines and dotted lines in these figures represent the average spectrum correlation coefficients between test data and training data with defect size of 0.007 inches and 0.021 inches, respectively. Correct classification results can be achieved by proposed method with the signal based triangular SE. The proposed method is also very useful in identifying the level of fault severity. From Figure 16(b), it can be observed that the morphology spectrums of outer race fault located at 6:00 with different defect sizes are very similar, demonstrating that the correlation analysis on morphology spectrum with flat SE is not appropriate for classifying the level of outer race fault severity. Detection results of data set $\mathrm{C}$ show that the signal based triangular SE performs much better than flat SE in detection of bearing faults with different defect sizes.

\section{Conclusions}

A signal based triangular SE and associated methodology are proposed in this study. This proposed SE is applied to MM closing operation for signal feature extraction. Consequently morphology spectrum can be then obtained from the processed signal by FFT. A correlation analysis on morphology spectrum is employed to give the final classification of bearing faults. This method is evaluated by practical vibration signal sets from bearings with inner race, ball, and outer race faults, respectively. All faults considered in CWRU can be identified without cases of misclassification. The ball fault which is usually difficult to identify by traditional methods can be detected accurately and clearly by the proposed approach. Compared with flat SE, the proposed method according to signal based triangular SE performs better in 
faults classification of bearing and can be used for identifying the location of outer race fault and the defect severity of bearing faults effectively.

\section{Conflict of Interests}

The authors declare that there is no conflict of interests regarding the publication of this paper.

\section{Acknowledgments}

Data used in this work is obtained from Bearing Data Center at Case Western Reserve University, http://csegroups.case .edu/bearingdatacenter/pages/welcome-case-western-reserve-university-bearing-data-center-website. The authors would like to thank Dr. Kenneth A. Loparo and Case Western Reserve University for their effort to make this data set available for all researchers. This work was supported by the National Natural Science Foundation of China (Grant no. 21076011) and Program for New Century Excellent Talents in University (Grant no. NCET-10-0210).

\section{References}

[1] C. Li, M. Liang, Y. Zhang, and S. Hou, "Multi-scale autocorrelation via morphological wavelet slices for rolling element bearing fault diagnosis," Mechanical Systems and Signal Processing, vol. 31, pp. 428-446, 2012.

[2] C. Li and M. Liang, "Continuous-scale mathematical morphology-based optimal scale band demodulation of impulsive feature for bearing defect diagnosis," Journal of Sound and Vibration, vol. 331, no. 26, pp. 5864-5879, 2012.

[3] N. Tandon and A. Choudhury, "Review of vibration and acoustic measurement methods for the detection of defects in rolling element bearings," Tribology International, vol. 32, no. 8, pp. 469-480, 1999.

[4] R. B. Randall and J. Antoni, "Rolling element bearing diagnostics-a tutorial," Mechanical Systems and Signal Processing, vol. 25, no. 2, pp. 485-520, 2011.

[5] B. Li, P.-L. Zhang, Z.-J. Wang, S.-S. Mi, and Y.-T. Zhang, "Gear fault detection using multi-scale morphological filters," Measurement, vol. 44, no. 10, pp. 2078-2089, 2011.

[6] P. D. McFadden and J. D. Smith, "Vibration monitoring of rolling element bearings by the high-frequency resonance technique-a review," Tribology International, vol. 17, no. 1, pp. 3-10, 1984.

[7] N. G. Nikolaou and I. A. Antoniadis, "Demodulation of vibration signals generated by defects in rolling element bearings using complex shifted Morlet wavelets," Mechanical Systems and Signal Processing, vol. 16, no. 4, pp. 677-694, 2002.

[8] Y.-T. Sheen, "On the study of applying Morlet wavelet to the Hilbert transform for the envelope detection of bearing vibrations," Mechanical Systems and Signal Processing, vol. 23, no. 5, pp. 1518-1527, 2009.

[9] Z. Peng, F. Chu, and Y. He, "Vibration signal analysis and feature extraction based on reassigned wavelet scalogram," Journal of Sound and Vibration, vol. 253, no. 5, pp. 1087-1100, 2003.

[10] Z. K. Peng, P. W. Tse, and F. L. Chu, "A comparison study of improved Hilbert-Huang transform and wavelet transform: application to fault diagnosis for rolling bearing," Mechanical Systems and Signal Processing, vol. 19, no. 5, pp. 974-988, 2005.

[11] B. Li, P.-L. Zhang, Z.-J. Wang, S.-S. Mi, and D.-S. Liu, "A weighted multi-scale morphological gradient filter for rolling element bearing fault detection," ISA Transactions, vol. 50, no. 4, pp. 599-608, 2011.

[12] N. E. Huang, Z. Shen, S. R. Long et al., "The empirical mode decomposition and the Hubert spectrum for nonlinear and non-stationary time series analysis," Proceedings of the Royal Society A: Mathematical, Physical and Engineering Sciences, vol. 454, no. 1971, pp. 903-995, 1998.

[13] A.-O. Boudraa and J.-C. Cexus, "EMD-based signal filtering," IEEE Transactions on Instrumentation and Measurement, vol. 56, no. 6, pp. 2196-2202, 2007.

[14] J. Cheng, D. Yu, and Y. Yang, "Application of support vector regression machines to the processing of end effects of HilbertHuang transform," Mechanical Systems and Signal Processing, vol. 21, no. 3, pp. 1197-1211, 2007.

[15] P. Maragos and R. W. Schafer, "Morphological filters-part I: their set-theoretic analysis and relations to linear shift invariant filters," IEEE Transactions on Acoustics, Speech, and Signal Processing, vol. 35, no. 8, pp. 1153-1169, 1987.

[16] G. Matheron, Random Sets and Integral Geometry, John Wiley \& Sons, New York, NY, USA, 1975.

[17] J. Serra, Image Analysis and Mathematical Morphology, Academic Press, New York, NY, USA, 1982.

[18] A. Hu, G. Tang, and L. An, "De-noising technique for vibration signals of rotating machinery based on mathematical morphology filter," Chinese Journal of Mechanical Engineering, vol. 42, no. 4, pp. 127-130, 2006.

[19] H. Li and D.-Y. Xiao, "Fault diagnosis using pattern classification based on one-dimensional adaptive rank-order morphological filter," Journal of Process Control, vol. 22, no. 2, pp. 436449, 2012.

[20] J. Wang, G. Xu, Q. Zhang, and L. Liang, "Application of improved morphological filter to the extraction of impulsive attenuation signals," Mechanical Systems and Signal Processing, vol. 23, no. 1, pp. 236-245, 2009.

[21] L. Zhang, D. Yang, J. Xu, and Z. Chen, "Approach to extracting gear fault feature based on mathematical morphological filtering," Chinese Journal of Mechanical Engineering, vol. 43, no. 2, pp. 71-75, 2007.

[22] N. G. Nikolaou and I. A. Antoniadis, "Application of morphological operators as envelope extractors for impulsive-type periodic signals," Mechanical Systems and Signal Processing, vol. 17, no. 6, pp. 1147-1162, 2003.

[23] Y. Dong, M. Liao, X. Zhang, and F. Wang, "Faults diagnosis of rolling element bearings based on modified morphological method," Mechanical Systems and Signal Processing, vol. 25, no. 4, pp. 1276-1286, 2011.

[24] L. Zhang, J. Xu, J. Yang, D. Yang, and D. Wang, "Multiscale morphology analysis and its application to fault diagnosis," Mechanical Systems and Signal Processing, vol. 22, no. 3, pp. 597610, 2008.

[25] S. Ouyang and J. Wang, "A new morphology method for enhancing power quality monitoring system," International Journal of Electrical Power and Energy Systems, vol. 29, no. 2, pp. 121-128, 2007.

[26] R. Hao, Z. Feng, and F. Chu, "Defects diagnosis and classification for rolling bearing based on mathematical morphology," in Proceedings of the 8th International Conference on Reliability, 
Maintainability and Safety (ICRMS '09), pp. 817-821, Chengdu, China, July 2009.

[27] K. A. Loparo, "Bearings vibration data set, Case Western Reserve University," http://csegroups.case.edu/bearingdatacenter/pages/download-data-file.

[28] W. Zhang, X. Zhou, and Y. Lin, "Application of morphological filter in pulse noise removing of vibration signal," in Proceedings of the 1st International Congress on Image and Signal Processing (CISP '08), pp. 132-135, Sanya, China, May 2008.

[29] W. Sun, G. A. Yang, Q. Chen et al., "Fault diagnosis of rolling bearing based on wavelet transform and envelope spectrum correlation," Journal of Vibration and Control, 2012.

[30] Q. Chen, Z. W. Chen, W. Sun et al., "A new structuring element for multi-scale morphology analysis and its application in rolling element bearing fault diagnosis," Journal of Vibration and Control, 2013.

[31] N. Sawalhi, R. B. Randall, and H. Endo, "The enhancement of fault detection and diagnosis in rolling element bearings using minimum entropy deconvolution combined with spectral kurtosis," Mechanical Systems and Signal Processing, vol. 21, no. 6, pp. 2616-2633, 2007.

[32] C. Li and J. Cheng, "Mathematical morphological filter and its application in removing noises in vibration signal," in Proceedings of the 8th International Conference on Electronic Measurement and Instruments (ICEMI '07), vol. 4, pp. 101-104, Xian, China, August 2007.

[33] R. Hao and F. Chu, "Morphological undecimated wavelet decomposition for fault diagnostics of rolling element bearings," Journal of Sound and Vibration, vol. 320, no. 4-5, pp. 1164-1177, 2009. 

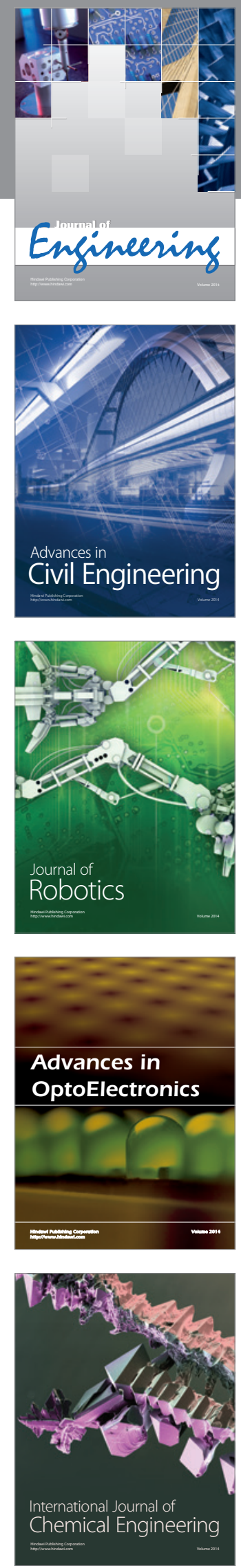

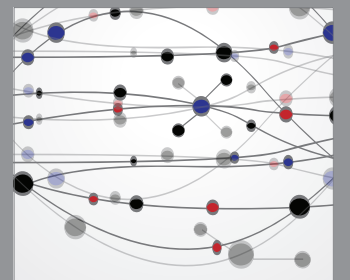

The Scientific World Journal
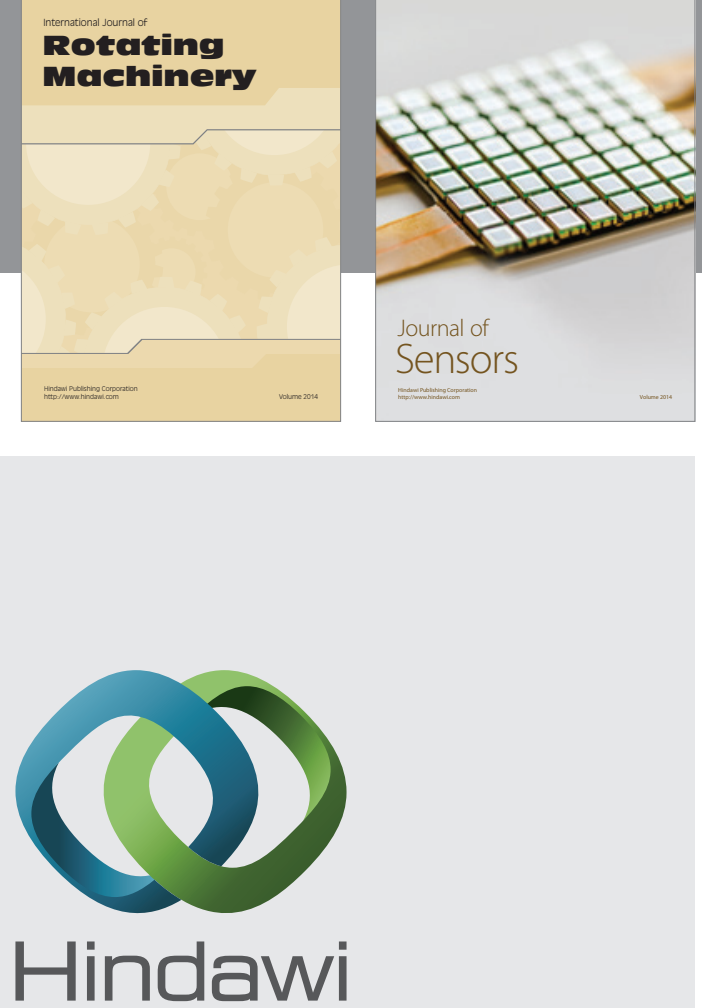

Submit your manuscripts at http://www.hindawi.com
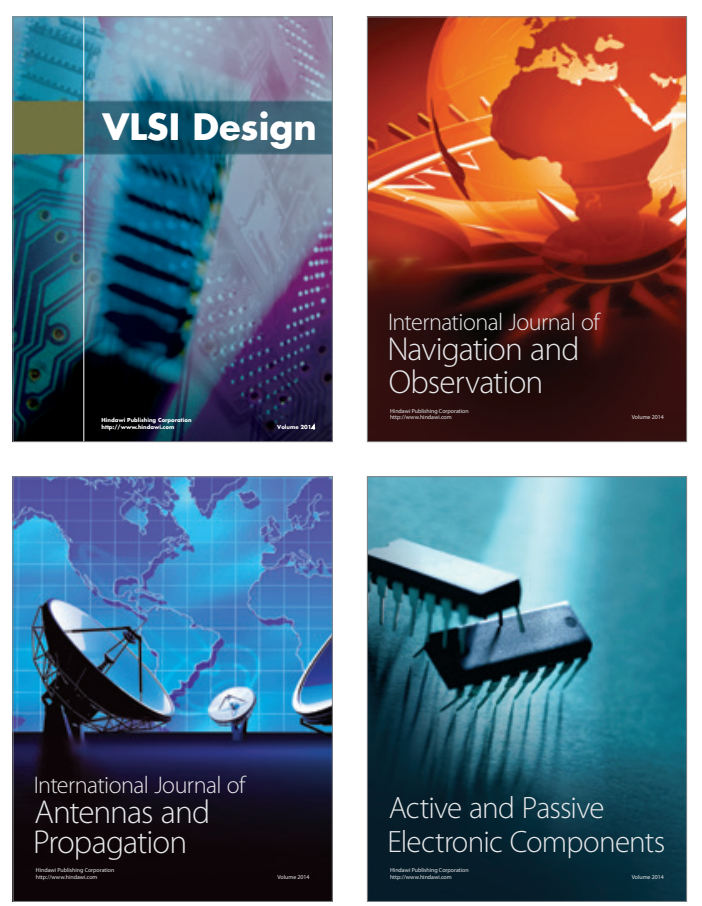
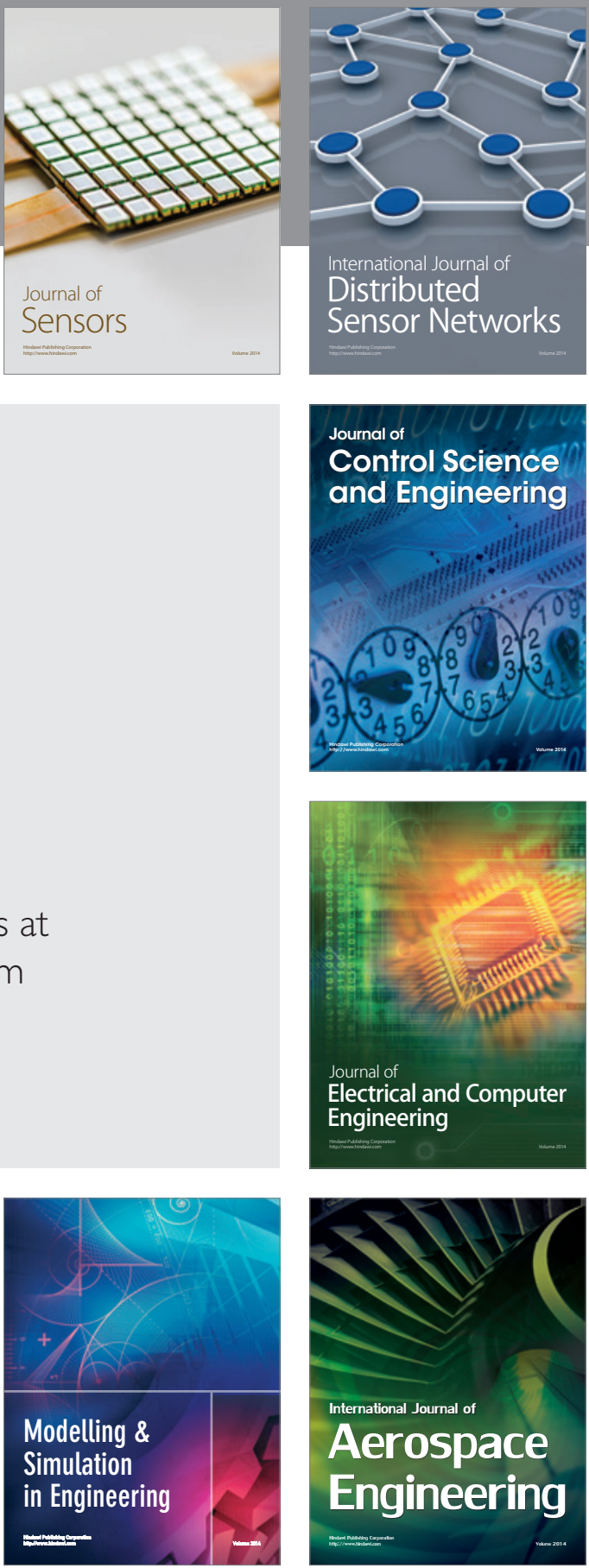

Journal of

Control Science

and Engineering
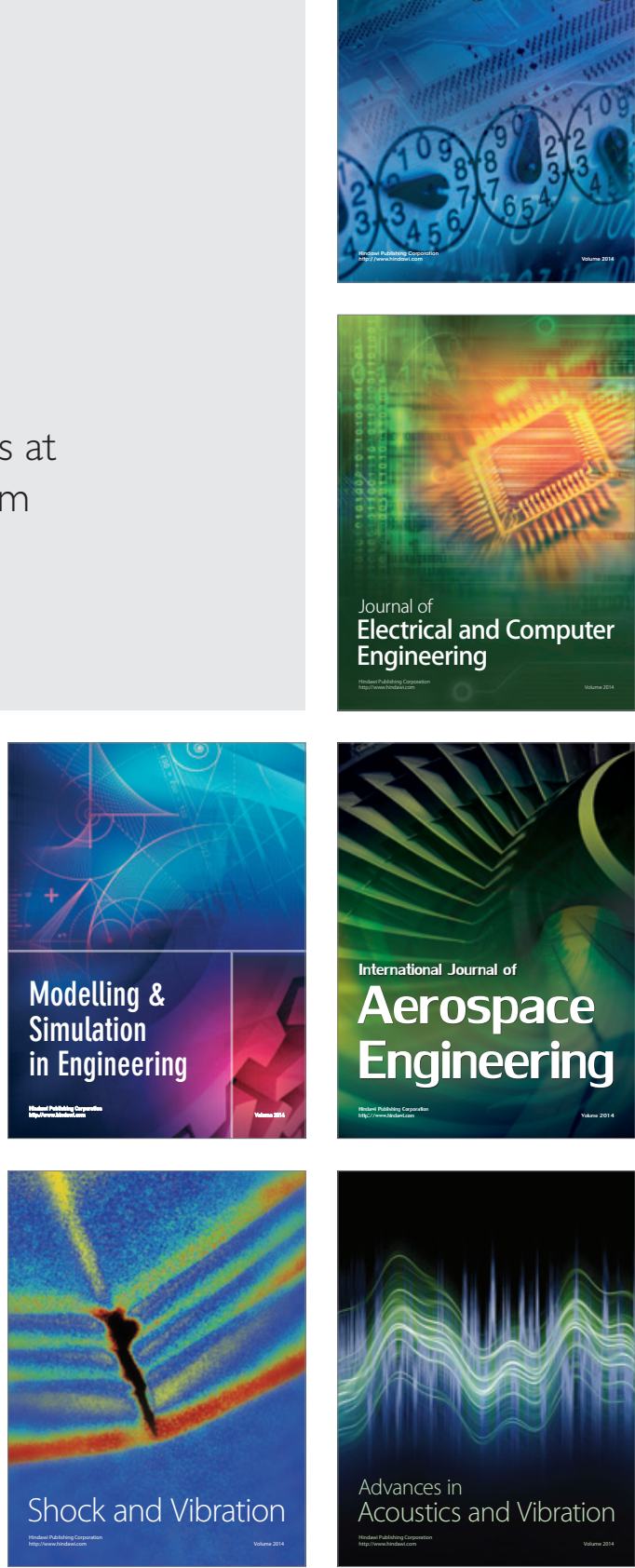